\title{
Modulation signal bispectrum with optimized wavelet packet denoising for rolling bearing fault diagnosis
}

\author{
Junchao Guo ${ }^{1}$, Zhanqun $\mathrm{Shi}^{1}$, Dong Zhen ${ }^{1, *}$, Zhaozong Meng ${ }^{1}$, Fengshou $\mathrm{Gu}^{2}$, Andrew. D. Ball ${ }^{2}$ \\ ${ }^{1}$ Tianjin Key Laboratory of Power Transmission and Safety Technology for New Energy Vehicles, School of \\ Mechanical Engineering, Hebei University of Technology, Tianjin, 300401, China. \\ ${ }^{2}$ Centre for Efficiency and Performance Engineering, University of Huddersfield, Huddersfield, HD1 3DH, UK. \\ ${ }^{*}$ Corresponding author. E-mail address: d.zhen@hebut.edu.cn (D. Zhen).
}

\begin{abstract}
Transient impulses caused by local faults are critical informative indicators for rolling element bearing fault diagnosis. The methods for accurately extracting transient impulses while suppressing strong background noise and interference components have received extensive studies. In this paper, a novel fault diagnosis scheme based on optimized wavelet packet denoising (WPD) and modulation signal bispectrum (MSB) is proposed, which takes advantage of the transient impulse enhancement of WPD and the demodulation ability of MSB to diagnose bearing faults more accurately. Firstly, the measured signals are decomposed into a series of time-frequency subspaces using wavelet packet transform. An optimal threshold value is selected based on the proposed threshold criterion by considering unbiased autocorrelation of envelope and Gini index (GI) of the transient impulses. Subsequently, the subspaces are denoised by the WPD with the optimized threshold value, and the master subspaces that containing the fault related transient impulses are selected based on the GI indicator. Finally, the MSB is utilized to further purify the signal and extract the modulation components contained in the transient impulses, and the suboptimal MSB slices are selected based on the characteristic frequency intensity coefficient (CFIC). The MSB detector is then obtained by averaging the suboptimal MSB slices to determine the type of the bearing faults. The proposed WPD-MSB is validated based on the simulation and experimental studies. Compared with the variational mode decomposition and Teager energy operator (VMD-TEO), fast kurtogram (FK) as well as conventional MSB, the proposed WPD-MSB method has superior performance in extracting the fault feature of the incipient defects on different bearing components.
\end{abstract}

Keywords: Optimized wavelet packet denoising; Modulation signal bispectrum; Unbiased autocorrelation; Gini index; Rolling element bearing. 


\section{Introduction}

Rolling element bearings are the heart of almost all rotating machines, and their health status is important for the normal operation of the entire transmission system. Due to the harsh environments and complex working conditions, faults occurring in bearings may lead to catastrophic accidents and expensive production losses. ${ }^{1-3}$ Thus, effective and timely diagnosis of bearing faults is of great significance to ensure the safe operation and reliability of machinery systems. When a local fault occurs in the bearing, transient impulses are extremely weak and easily buried in strong background noise and interference components due to the interference of the complicated transmission path and working environment. ${ }^{4-5}$ Therefore, it is highly important to accurately extract transient impulses from vibration signals with background noise and interference components, especially for the incipient fault detection of rolling element bearing.

Wavelet transform (WT), as a competitive method for transient impulses identification, has been drawn great attention in theoretical analysis and engineering applications in the field of fault detection. ${ }^{6-8}$ However, the WT cannot divide the high frequency band that contained most of the mechanical fault modulation information. To improve the analysis ability for high frequency band of WT, the wavelet packet transform (WPT) is developed, which not only produces more detailed frequency bands, but also enhances the ability of obtaining the relevant fault information from the measured signal. In recent years, WPT has received widespread attention for denoising in the field of rotating machinery fault detection and condition monitoring. ${ }^{9-12}$ For instance, Wang et al. ${ }^{9}$ presented a novel scheme that combines wavelet packet denoising (WPD) and random forests to obtain weak transient impulse signals with strong background noise and identify faults in rolling bearings. Xing et al. ${ }^{10}$ proposed a new approach based on the optimal WPD and artificial neural network (ANN) for detecting human perceptional characteristics. Liu et al. ${ }^{11}$ introduced an improved WPD approach based on noise variance estimation to extract signal features, and proved the performance of the algorithm by simulation and experimental studies. Li et al. ${ }^{12}$ applied an undecimated lifting WPD and Shannon entropy-improved adaptive line enhancer to detect rail failures effectively. These studies demonstrated that the WPD can be considered as a splendid signal representation approach for identifying weak transient impulse signals from strong background noise. However, the effective applications of WPD strictly depend on the wavelet decomposition level, threshold function, mother wavelet and threshold criterion.

At present, a large number of investigations reported in the literatures have focused on improving the performance of WPD for transient impulses extraction which can be divided into four categories according to the above-mentioned parameters. Among them, wavelet decomposition level, mother wavelet and threshold function are three noticeable topics. Regarding to the wavelet decomposition level, a few researchers have developed the appropriate parameters based on the user's experience, ${ }^{13}$ 
white noise test method, ${ }^{14}$ energy, ${ }^{15}$ frequency range, ${ }^{16}$ signal to noise ratio (SNR) ${ }^{17}$ and Shannon entropy-based criterion. ${ }^{18}$ Regarding to the mother wavelet, various typical mother wavelets have been devoted to machine fault diagnosis, for instance, Biorthogonal (biorNr.Nd), ${ }^{19}$ Morlet (morl), ${ }^{20}$ Coiflet (coifN), ${ }^{21}$ Daubechies (dbN), ${ }^{22}$ Meyer (meyr) ${ }^{23}$ and SymletsA (symN), ${ }^{24}$ etc. For these wavelet families, Meyer is the most attractive one for WPD in bearing fault detection, because it has the excellent performance of time and frequency localization and is similar to the transient impulse signal of a bearing in the wavelet families. ${ }^{25}$ Regarding to the threshold function, researchers have proposed various methods, such as hard and soft threshold functions, ${ }^{26}$ power function, ${ }^{27}$ semi-soft function, ${ }^{28}$ and sin threshold function. ${ }^{29}$ In addition, Mishra et al. ${ }^{30}$ proposed a sigmoid threshold function to extract the transient impulses from vibration signal under slow operation. Xu et al. ${ }^{13}$ introduced a continuous derivable threshold function and gradient descent to extract the weak echo signal of the laser radar.

It is worth noting that the threshold criterion is also one of the key factors to improve the performance of WPD, although it is rarely discussed so far. Recently, a few specific criterions have been proposed to select the optimal threshold value. For instance, Feng et al. ${ }^{14}$ introduced the mean square error (MSE) criterion in selecting the optimal threshold value. Liu et al. ${ }^{31}$ put forward noise variance estimation to optimize the optimal threshold value. Although the above method can obtain the best optimal threshold value to achieve a good noise reduction effect, it still requires a large number of iterative calculations, which leads to low calculation efficiency. Subsequently, Xu et al. ${ }^{32}$ adopted particle swarm optimization (PSO) and Han et al. ${ }^{33}$ proposed quantum genetic algorithm (QGA) to pursue the optimal threshold value, but PSO and QGA are extremely limited by the definition of their fitness function. To solve the above issues, this paper puts forward a novel threshold criterion based on the unbiased autocorrelation of envelope and Gini index (GI), which can accurately determine the optimal threshold value of sifting iterations. This criterion can effectively characterize the transient impulses in the measured signal, so it is regarded as a novel indicator to determine the optimal threshold value. However, there are still deterministic interference components and nonlinear modulation components after wavelet threshold denoising, and the denoised signal still faces contamination with in-band noise. Therefore, further procedures are needed to diagnosis the fault signature information in an accurate manner.

Modulation signal bispectrum (MSB) is a typical signal demodulation algorithm that has been widely applied for fault feature extraction. It can effectively extract modulation components by means of restraining background noise and interference components. With the above advantages, MSB has caused widespread concern in rotating machinery fault detection. ${ }^{34-38}$ Zhang et al. ${ }^{34}$ put forward a new approach to detect gear wear using the MSB for timely prediction and maintenance. Tian et al. ${ }^{35}$ demonstrated that MSB-based method is capable of generating more robust diagnosis results for 
detecting common bearing faults in a series of representative scenarios. Guo et al ${ }^{36}$ put forward a novel planetary gearbox fault detection scheme using enhanced average (EAVG) filter and MSB. Guo et al. ${ }^{37}$ explored an enhanced MSB based on autoregressive (AR) modeling, which effectively solved the weakness that MSB cannot effectively deal with non-Gaussian noise. Xu et al. ${ }^{38}$ proposed a phase linearisation-based MSB (PL-MSB) to process the cyclostationary signal for bearing fault diagnosis. The above-mentioned studies have shown that MSB has superior performance in terms of demodulation and noise reduction. However, the selection of suboptimal MSB slices relies on user experience, which enormously restricts the applications of the MSB and may lead to incorrect detection results. Moreover, there is still a problem for the MSB in dealing with non-stationary vibrations, that is, it enhances the features of other interference components while enhancing the fault features.

Based on the above investigation, a novel scheme combining WPD and MSB is developed for rolling element bearing fault detection. Firstly, the vibration signal is decomposed into different time-frequency subspaces using WPT. A new threshold criterion considering two characteristics, namely, unbiased autocorrelation of envelope and Gini index (GI), which is developed for determining the optimal threshold value to further improve the denoising effect of each subspace. Secondly, the master subspaces are selected according to the principle of GI to obtain the transient impulses from bearing vibration signal. Then, the MSB is utilized to demodulate the modulation components in the impulse components, and the suboptimal MSB slices are selected based on the characteristic frequency intensity coefficient (CFIC) to obtain the MSB detector. Finally, the fault identification is inferred by the occurrence of fault defect frequencies in the MSB detector.

The remainder of the paper is organized as follows. Section, "Transient signal extraction based wavelet packet denoising" introduces the basic principle of the WPD for transient impulse signal extraction. Section, "Modulation signal bispectrum" presents the fundamental theories of the MSB. Section, "The proposed fault diagnosis scheme" introduces the detailed procedure of the proposed scheme based on WPD and MSB. Section, "Simulation analysis" applies the WPD-MSB to the simulated signal and compares it with the conventional MSB. Section, "Experiment validation" applies the WPD-MSB to vibration signals acquired from a planetary gearbox bearing with inner race and a motor bearing outer race fault. Finally, the conclusions are drawn in section "Conclusion".

\section{Transient signal extraction based wavelet packet denoising}

\section{Wavelet packet transform}

Wavelet packet transform (WPT) is an enhanced weak transient identification approach, which can separate the original signal into different time-frequency subspaces at any time-frequency resolution. ${ }^{39}$ The wavelet pack function $W_{j, k}^{n}(t)$ is defined as: 


$$
W_{j, k}^{n}(t)=2^{j / 2} W^{n}\left(2^{j} t-k\right)
$$

where $j, k$ and $n\left(n=0,1, \ldots, 2^{j}-1\right)$ are the scale, translation parameter and oscillation parameter, respectively. When $j=k=0$, the first two scale function $\Phi(t)$ and mother wavelet function $\Psi(t)$ satisfy the two-scale equation in multi-resolution analysis and they are defined as follow:

$$
\begin{aligned}
& W_{0,0}^{0}(t)=\Phi(t) \\
& W_{0,0}^{1}(t)=\Psi(t)
\end{aligned}
$$

The remaining wavelet pack functions $W^{n}(t)$ for $n>1$ can be obtained from the scale function and mother wavelet function as:

$$
\begin{aligned}
W^{2 n}(t) & =\sqrt{2} \sum_{k} h(k) W_{1, k}^{n}(2 t-k) \\
W^{2 n+1}(t) & =\sqrt{2} \sum_{k} g(k) W_{1, k}^{n}(2 t-k)
\end{aligned}
$$

where $h(k)=1 / \sqrt{2}\langle\varphi(t), \varphi(2 t-k)\rangle$ stands the low-pass filter and $g(k)=1 / \sqrt{2}\langle\psi(t), \psi(2 t-$ $k)\rangle$ represents the high-pass filter. The two filters are also mutually orthogonal with the relationship $g(k)=(-1)^{k} h(1-k)$. The wavelet packet (WP) coefficient $Q_{j, k}^{n}$ is acquired using the inner product between the signal $s(t)$ and the WP functions $W_{j, k}^{n}$, which can be defied as:

$$
Q_{j, k}^{n}=\left\langle s(t), W_{j, k}^{n}(t)\right\rangle=\int_{-\infty}^{+\infty} s(t) W_{j, k}^{n}(t) d t
$$

where $Q_{j}^{n}(k)$ represents the $n^{\text {th }}$ set of wavelet packet coefficients of the $j^{\text {th }}$ scale parameter.

Mathematically, for a discrete-time signal $x(t)$ with a data length of $M\left(M=2^{n_{0}}\right)$, the reconstructed signal of wavelet coefficients at each node is represented by $\left\{P_{j}^{n}(k), k=1,2, \ldots, 2^{n_{0}}\right\}$. Assuming that the WP node $(j, n)$ represents a container with an area of $2^{n_{0}}$, the waveform feature (WF) distributed in the container is expressed using $P_{j}^{n}$. The WF space for the $(j, n)$ th level is defined as $^{40}$ :

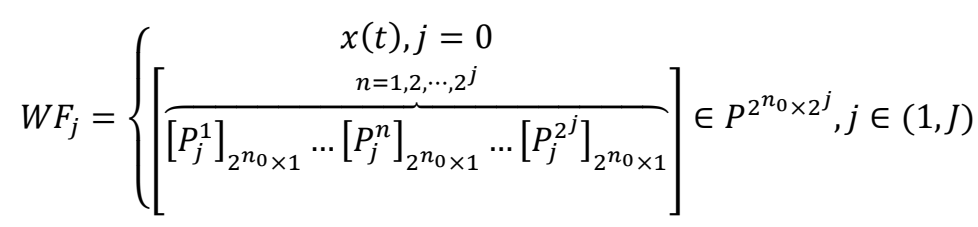

Putting the $W F_{j}$ from level 0 to $J$ together, the $W F$ is defined as:

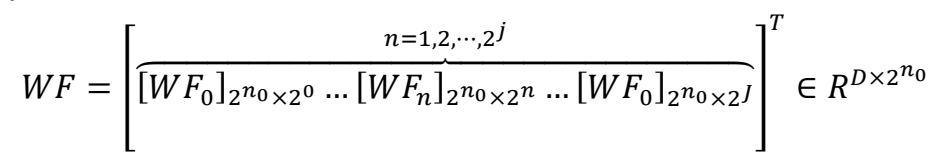

where $D=2^{0}+2^{1}+\cdots+2^{J}$, which indicates the dimension of the $W F$ space.

\section{Proposal of the optimized WPD}

\section{Optimization of threshold value for WPD}

The performance of WPD relies on four aspects: wavelet decomposition level, threshold function, mother wavelet and threshold criterion. It is necessary to make reasonable choice in the four aspects to effectively remove random noise and interference components to enhance the transient impulse 
components. The first three aspects, including the wavelet decomposition level, mother wavelet and threshold function have been investigated in the previous researches, ${ }^{13,21}$ while the potentiality of threshold criterion deserves further studies. This investigation will focus on the influence of threshold criterion on the transient impulses extraction using WPD, and then put forward a novel threshold criterion that determines the optimal threshold value of sifting iterations by considering the trade-off between enhanced the signal-to-noise ratio (SNR) and reserved signal energy. In addition, the existing approaches of wavelet decomposition level, ${ }^{13}$ mother wavelet ${ }^{23}$ and threshold function ${ }^{26}$ are applied.

Threshold criterion is a key factor for WPD in suppressing background noise to extract transient impulse signals. A large threshold value will lead to the loss of valid information, while a small threshold value will affect the noise reduction effect. In other words, a reasonable threshold value needs to ensure a trade-off between signal energy retention and SNR enhancement. Thus, it is very important to explore an effective threshold criterion to select the most optimal threshold value. Unbiased autocorrelation is an effective technique for extracting periodic impulse components in vibration signals. ${ }^{41-42}$ For a vibration signal $y(t)$ composed of the periodic impulse signal $x(t)$ and noise $n(t)$, the unbiased autocorrelation of envelope is calculated by: ${ }^{43}$

$$
\hat{R}_{Y Y}(\tau)=\frac{1}{N-q} \sum_{i=1}^{N-q} Y\left(t_{i}\right) Y\left(t_{i}+\tau\right)
$$

$$
=\frac{1}{N-q} \sum_{i=1}^{N-q}\left[X\left(t_{i}\right)+N\left(t_{i}\right)\right]\left[X \left(t_{i}+\right.\right.
$$

$\left.\tau)+N\left(t_{i}+\tau\right)\right]$

$\hat{R}_{N X}(\tau)+\hat{R}_{N N}(\tau)$

$$
=\hat{R}_{X X}(\tau)+\hat{R}_{X N}(\tau)+
$$

where $Y(t)$ means the envelope of the signal $y(t), \tau=q / f_{s}$ means the delay factor and $q=$ $0, \ldots, N-1$, and $f_{s}$ is the sampling frequency. Being random, white Gaussian noise $\hat{R}_{N N}(\tau)=0$. In addition, the periodic impulse signal $x(t)$ and background noise $n(t)$ are independent, so the cross-correlation between them is omitted: $\hat{R}_{X N}(\tau)=0 \hat{R}_{N X}(\tau)=0$. The unbiased autocorrelation of the envelope in Eq. (9) is redefined as:

$$
\hat{R}_{Y Y}(\tau)=\hat{R}_{X X}(\tau)
$$

This shows that the application of unbiased autocorrelation can significantly remove the background noise in vibration signal while maintaining the signal energy greatly.

Gini index (GI) was originally proposed by Corrado Gini to assess the economic income gap, and it has been regarded as a sparse indicator. Recently, Miao et al. ${ }^{44}$ applied GI to replace the kurtosis criterion to improve the performance of Kurtogram in characterizing transient impulses. Wang et al. ${ }^{45}$ used GI to quantitatively measure the sparsity of time-frequency representation, and through experimental analysis proved that GI has a powerful ability to reveal transient impulses in vibration signal and has better performance in resisting random impulses. Wang et al. ${ }^{46}$ have proposed a MSB-GI to indicate gradual changes in bearing internal clearances for a stable and bounded monitoring result. 
Because of the robustness and bounded property of GI, it is adopted in this study to show the components of transient impulses, which is defined as follows:

$$
G I=1-2 \sum_{n=1}^{N} \frac{x_{(n)}}{\|x\|_{1}}\left[\frac{N-n-\frac{1}{2}}{N}\right]
$$

where the vector $x=\left[x_{(1)}, x_{(2)}, \ldots, x_{(N)}\right]$ and the elements are sorted, $x_{(1)} \leq x_{(2)} \leq \cdots \leq x_{(N)}$. In addition, $\|x\|_{1}$ stands the $L_{1}$ norm of $x$. Taking into account the energy retention and impulse enhancement, a novel wavelet threshold criterion $F_{m}$ is proposed as:

$$
F_{m}=\widehat{N R}_{X X}\left(\Delta_{m}\right)-G I\left(\Delta_{m}\right)
$$

where the threshold value is $\Delta_{m}=m \delta, \delta=\max \left(\operatorname{abs}\left(Q_{j, k}^{n}\right)\right) / M$, and $m(m=1,2, \cdots, M)$ is the iteration number of wavelet threshold denoising, where $M$ can be a small number (for example, 10 in the case of Fig. 4) for quickly find the suboptimal region. As shrinking wavelet coefficient will result in reduction in signal energy but increase in GI, it is straightforward to determine an optimal threshold values $\Delta_{m}^{o}$ can be determined by Eq. (13):

$$
\widehat{N R}_{X X}\left(\Delta_{m}^{o}\right)-G I\left(\Delta_{m}^{o}\right)=0
$$

where $\widehat{N R}_{X X}=\hat{R}_{X X} / \max \left(\widehat{R}_{X X}\right)$ represents the normalized form of $\hat{R}_{X X}$, i.e. normalized wavelet packet energy (NWPE). The reason for normalizing $\hat{R}_{X X}$ is that $\hat{R}_{X X}$ is a dimensional unit, but the value of GI is bounded within the range of $[0,1]$. The optimization process of the threshold value can be summarized as the flowchart in Fig. 1, which can be elaborated as follows:

Step 1: Perform binary WPT on the original signal to obtain a series of time-frequency subspaces.

Step 2: For each search threshold value $\Delta_{m}$, implement wavelet threshold denoising on the time-frequency subspaces.

Step 3: Calculate the normalized wavelet packet energy (NWPE) matrix in the denoising subspaces using Eq. (14):

$$
\left\{\begin{array}{c}
W P E_{j}\left(\Delta_{m}\right)=\sum_{m=1}^{M} \sum_{n=1}^{N}\left[W F_{j}^{n}\left(\Delta_{m}\right)\right]^{2} \\
N W P E_{j}\left(\Delta_{m}\right)=W P E_{j}\left(\Delta_{m}\right) / \max \left(W P E_{j}\left(\Delta_{m}\right)\right)
\end{array}\right.
$$

where $j$ indicates the layer of the tree, $n=1, \ldots, 2^{j}$ and $N_{j}$ are the node and length in layer $j$, respectively.

Step 4: Select the master subspaces, that is, find the subspaces with local peaks from $N W P E_{j}\left(\Delta_{m}\right)$.

Step 5: Obtain the unbiased autocorrelation of envelope and GI values of the master subspaces by iterating $M$ times from Step 2 to 4 , and these values are substituted into the Eq. (12) to formulate $F_{m}$ 
and determine a coarse $\Delta_{m}^{o}$.

Step 6: Refine the threshold value $\Delta_{m}$ around $F_{m}=0$ using the cubic spline interpolation method, and the obtained threshold value is taken as the optimal threshold value $\Delta_{m}^{o}$.

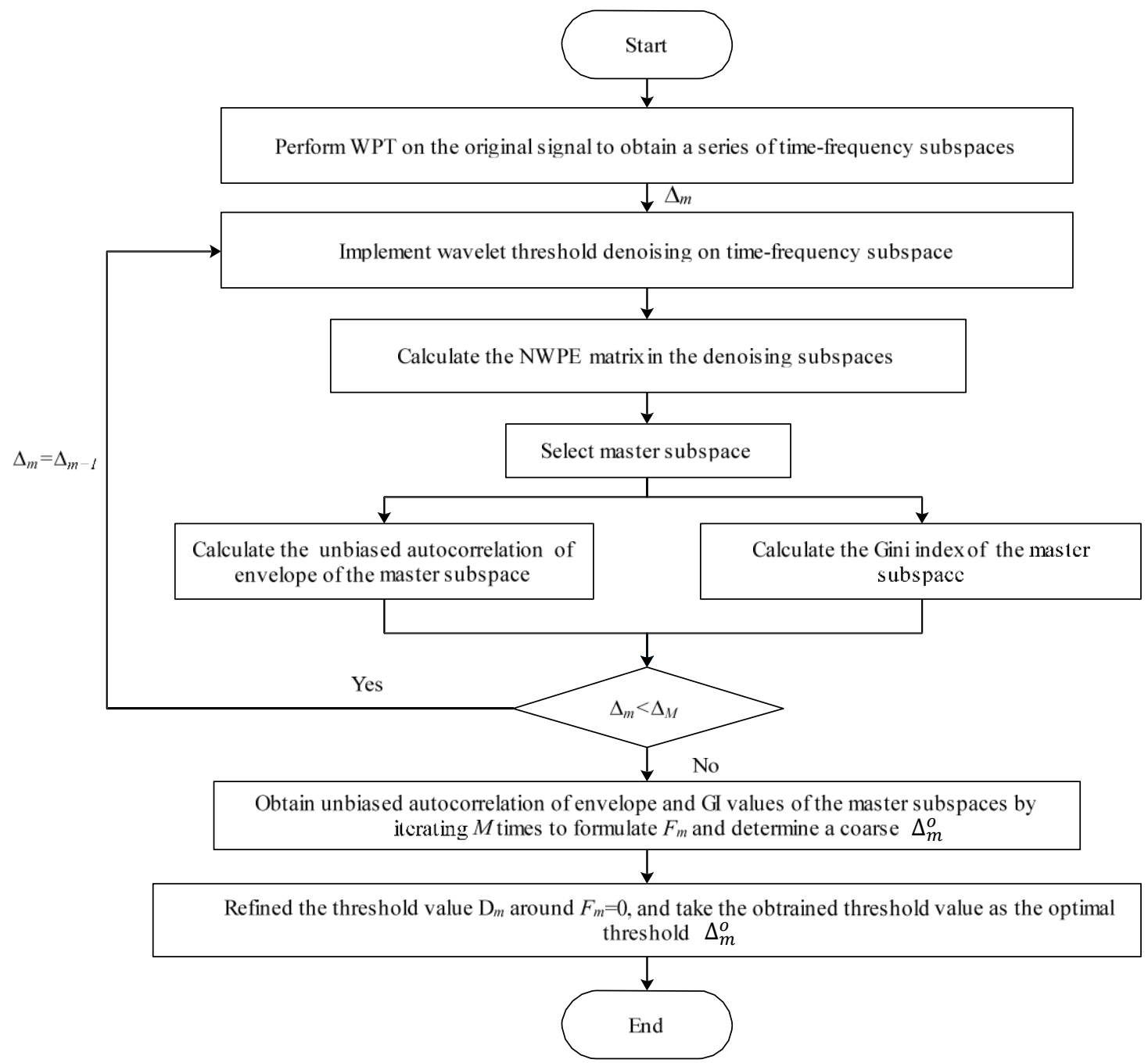

Fig. 1. The flowchart of threshold value optimization.

\section{Verification of optimal threshold value}

To verify that the threshold value $\Delta_{m}$ corresponding to $F_{m}=0$ is the optimal threshold value $\Delta_{m}^{o}$, a typical simulation fault model ${ }^{47-48}$ is used to simulate cyclostationary signals. The bearing outer race fault model consisting of $2 K+1$ fault impulses can be described as:

$$
x(t)=\sum_{k=-K}^{K} A_{k} e^{-\left[\zeta 2 \pi f_{n}\left(t_{i}\right)\right]} \cos \left[2 \pi f_{n}\left(t_{i}\right)\right] u\left(t_{i}\right)+n(t)
$$

where $A_{k}$ represents the amplitude of the $k$ th fault impulse, $K$ denotes the number of fault impulse, $\zeta$ represents the damping characteristic factor, $f_{n}$ indicates the excited resonance frequency, $u(t)$ means a unit step function, $t_{i}=t-\left(k T+\sum_{i=-K}^{k} \tau_{i}\right)$ in which $T$ stands for the fault impulse interval, 
$\tau_{i}$ denotes the influence of random slippage of the rolling elements, and serves as the $i$ th realization of a zero mean uniformly distributed random variable, with a standard deviation of $0.01 T \sim 0.02 T$, and $n(t)$ means Gaussian white noise. Table 1 lists the parameters applied in the simulated bearing signals. Meanwhile, the sampling frequency $F_{S}=96 \mathrm{kHz}$ and sampling number $N=960,000$. Fig. 2 illustrates the time randomness of the first 100 fault feature impulses for differing degrees of slippage $(0 \%, 0.4 \%$ and $2 \%$ ). Obviously, the deviation from the fault period will be greater when more random slippage occurs, and the vibration response involves more random components and less periodicity. As shown in Fig. 2(b), the spectral smearing effects become more serious as the degree of random slippage increases, which exhibit the typical characteristics of cyclostationary signals. This will cause the frequency components to be ambiguous and form a continuous spectrum. However, the response shows higher amplitudes with observable discrete sidebands for different slippage cases in the resonance band around $7 \mathrm{kHz}$. This shows that it is possible to extract the fault information once the randomness is suppressed adequately.

Table 1. Parameter of the simulated signal.

\begin{tabular}{cc}
\hline Parameter & Value \\
\hline$K$ & 350 \\
$\zeta$ & 0.03 \\
$f_{n}$ & $7000 \mathrm{~Hz}$ \\
$T$ & $1 / 70 \mathrm{~s}$ \\
\hline
\end{tabular}
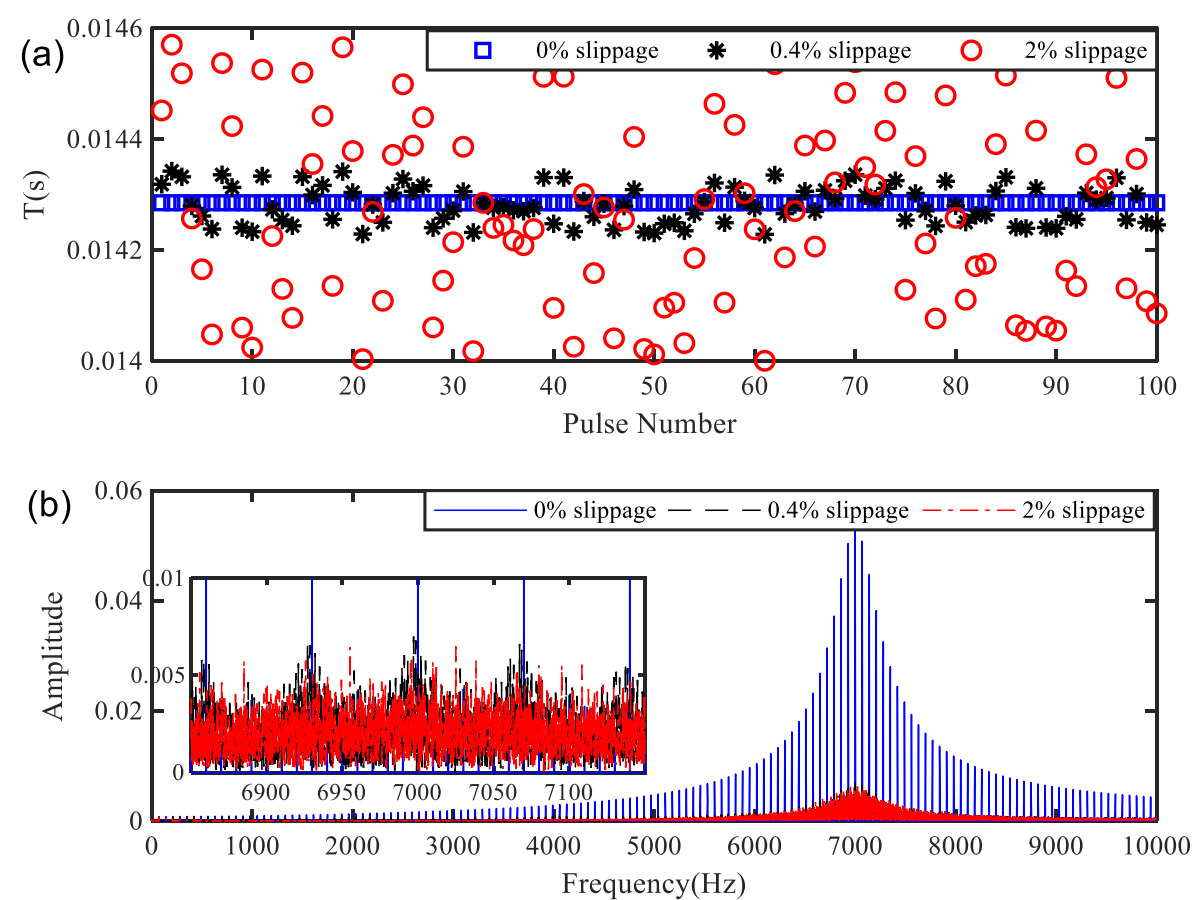

Fig. 2. Simulated signal of an outer race defect without added noise: (a) the time randomness of fault features impulses (b) the spectrum of (a).

To show the effectiveness of the proposed WPD method, the case with $2 \%$ random slippage is taken as basis for different levels of additive noises as shown in Fig. 3. The signal presented in point 
line in Fig. 3 illustrates the waveform and its spectrum of the simulated signal (with 2\% slippage) with additive noise $(\mathrm{SNR}=-10 \mathrm{~dB})$. And the solid line exhibited in Fig. 3 is the simulated signal (with $2 \%$ slippage) without noise. According to the flowchart of Fig. 1, the optimal threshold value $\Delta_{m}^{o}$ can be obtained with following detailed operations.
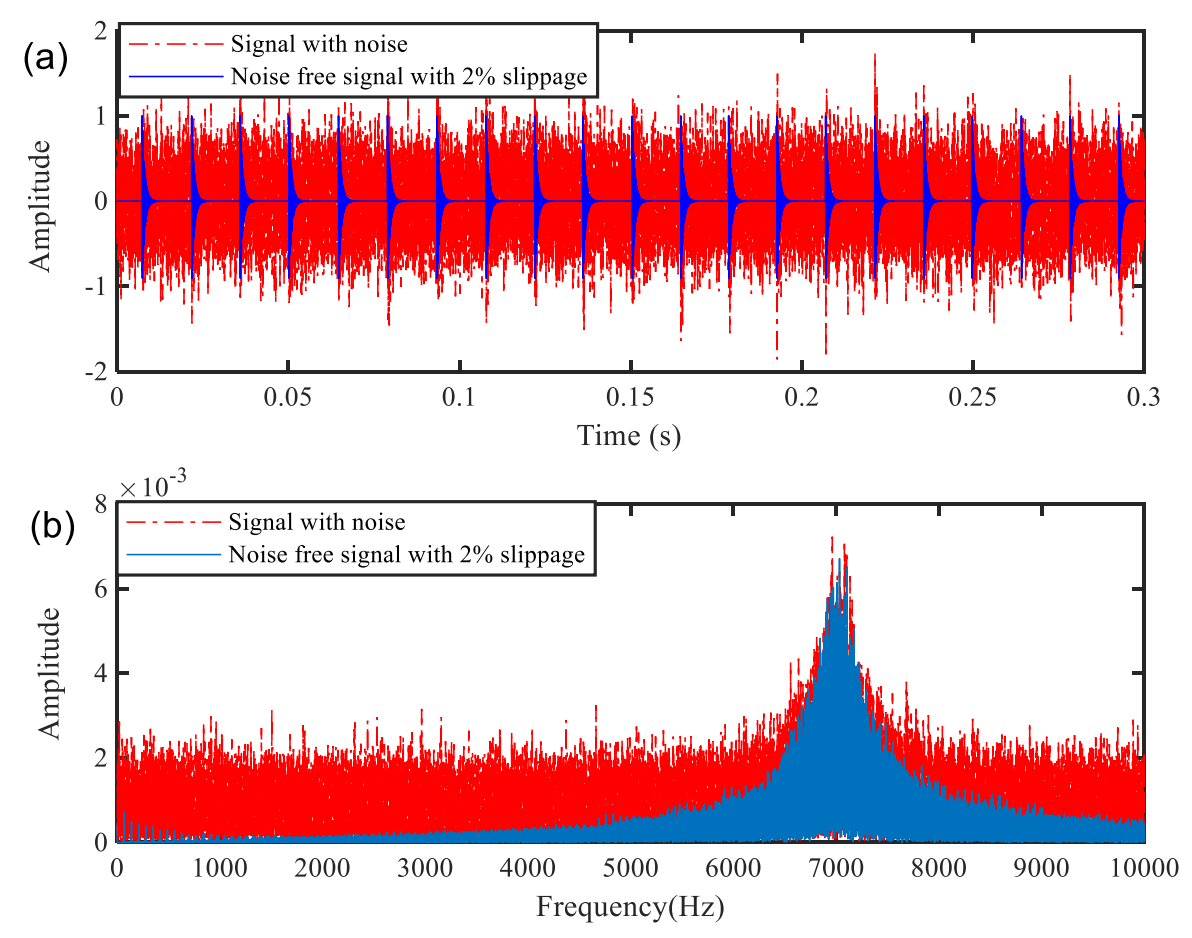

Fig. 3. Simulated signal of an outer race defect with the SNR of $-10 \mathrm{~dB}$ : (a) waveform with $2 \%$ random slippage; (b) spectrum of the (a).

Firstly, the simulated signal in Fig. 3(a) was decomposed into 32 time-frequency subspaces using Meyer wavelet. According to the maximal magnitude of wavelet coefficients, $\Delta_{m}$ was set as 0.1772 when $\mathrm{M}=7$ to calculate GI and NWPE at the threshold values in the amplitude range. A smaller $\Delta_{m}$ will have finer search results but with higher computing efforts. Based on simulation studies, it was found that $\mathrm{M}$ is set to 10 will be sufficient. Alternatively, the iteration can be also automatically terminated once it finds that GI is higher than NWPE. As shown in Fig. 4, the searching iteration can be stopped immediately after iteration 3. From Fig. 4, it can be seen that the optimal threshold values $\Delta_{m}^{o}$ can be determined as 0.2232 according to Eq. (13). Especially, both GI and NWPE exhibit monochromic trends with the threshold values, allowing the fast search to be fulfilled by the interpolation. 


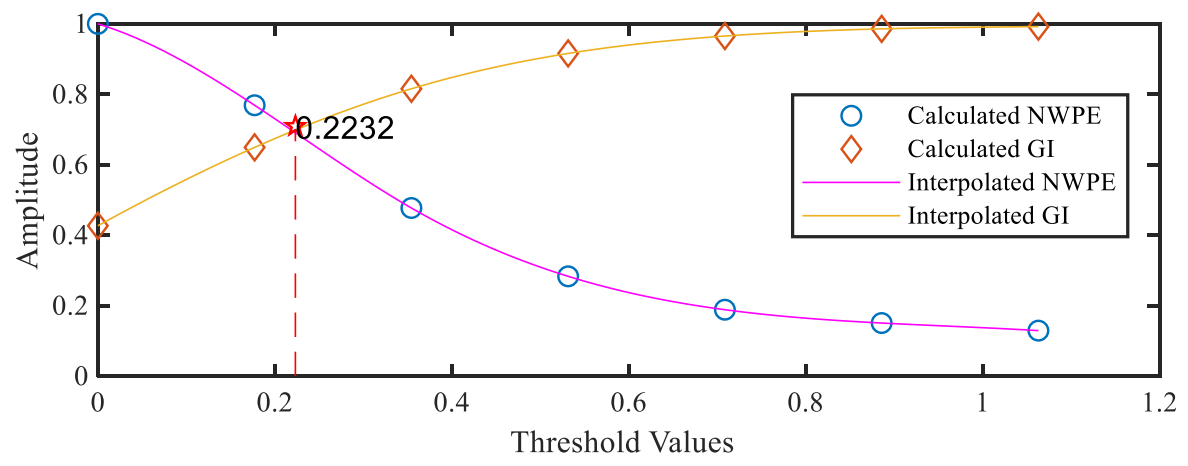

Fig. 4. Result of the threshold value optimization.

To confirm the effectiveness of the optimal threshold value, the GI values were also calculated for the spectrum at different threshold values. As shown in Fig. 5, the maximal GI value appears at 0.355. It shows that the spectrum at this value exhibit the sparsest characteristics. ${ }^{49}$ This also means that a higher threshold value is needed to achieve optimized spectrum. However, this higher threshold value can cause over-shrinkage to signal energy. In addition, there is only little tiny difference in GI value around the optimal threshold. Therefore, the smaller threshold value in Fig. 4 is taken as the optimal threshold value i.e. $\Delta_{m}^{o}=0.2232$ for this case.

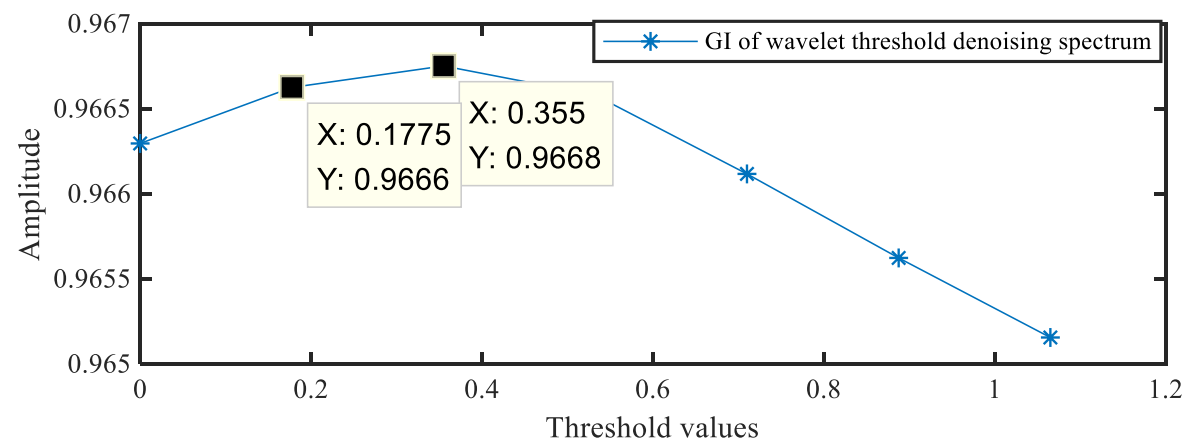

Fig. 5. The GI of wavelet threshold denoising spectrum under different threshold values.

Furthermore, the Universal threshold proposed by Donoho is the most commonly used method, ${ }^{26,50}$ which is applied to analyse the simulated signal in Fig. 3(a). The filtered signal using the Universal threshold was depicted in Fig. 6(b). It can be seen that the transient signal cannot be effectively revealed, and there is still a large amount of random noise compared with Fig. 6(a) obtained by the proposed optimal threshold. Therefore, it is verified that the proposed threshold scheme is more effective in enhancing transient impulse signals with strong background noise. 

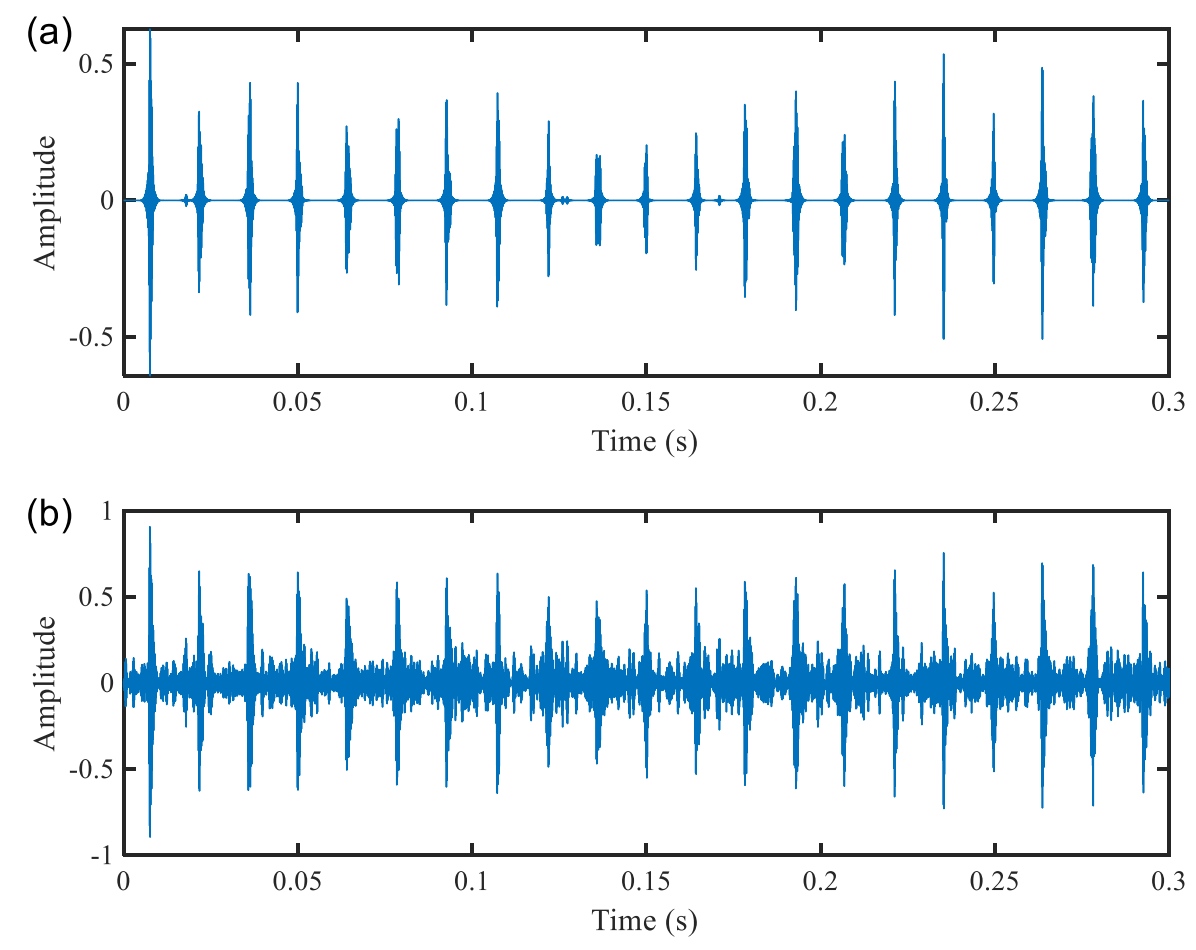

Fig. 6. Results of transient impulse signal by: (a) the proposed threshold criterion with optimal threshold value; (b) Universal threshold.

\section{Implementation of the optimized WPD}

The implementation of the optimized WPD can be summarized. Assuming that there is an observed signal $x_{i}$, which can be expressed as $^{32}$ :

$$
x_{i}=s_{i}+n_{i}(i=0,1, \ldots, N-1)
$$

where $s_{i}$ indicates the $i$ th raw signal, and $n_{i}$ represents the noise signal. The noise signal obeys the normal distribution, and its variance is constant. The optimized WPD algorithm can be implemented through the following three key steps:

(1) Wavelet packet decomposition. Select reasonable wavelet decomposition level and mother wavelet for WPT. Then decompose the signal $x_{i}$ into a series of time-frequency subspaces by WPT.

(2) Wavelet threshold denoising. Choose the reasonable threshold function and obtain the optimal threshold value according to Fig. 1, and implement them on the time-frequency subspaces.

(3) Wavelet packet reconstruction. Select the master subspaces containing fault related transient impulses based on the GI. Then, the inverse WPT is applied to the master subspaces to obtain the reconstructed signal. 


\title{
Modulation signal bispectrum
}

\author{
Brief review of $M S B$
}

The modulation signal bispectrum (MSB) method is a significant improvement over the conventional bispectrum for suppressing random noise and aperiodic components interference, and clearly reflecting the modulation components in the measured signal. ${ }^{35-36}$ For a discrete signal $x(t)$ with corresponding discrete Fourier transform (DFT) $X(f)$, the MSB can be formulated as follows:

$$
B_{M S}\left(f_{1}, f_{2}\right)=E<X\left(f_{1}+f_{2}\right) X\left(f_{1}-f_{2}\right) X^{*}\left(f_{1}\right) X^{*}\left(f_{1}\right)>
$$

where $B_{M S}\left(f_{1}, f_{2}\right)$ and $E<>$ indicate the bispectrum of signal $x(t)$ and expectation operator. The $f_{1}$ and $f_{2}$ represent the carrier frequency and modulating frequency, $\left(f_{1}+f_{2}\right)$ and $\left(f_{1}-f_{2}\right)$ denote the higher and lower sideband frequencies, respectively.

The whole phase of the MSB is defined as follow:

$$
\emptyset_{M S}\left(f_{1}, f_{2}\right)=\varnothing\left(f_{1}+f_{2}\right)+\emptyset\left(f_{1}-f_{2}\right)-\emptyset\left(f_{1}\right)-\emptyset\left(f_{1}\right)
$$

When two components $f_{1}$ and $f_{2}$ are in coupling, their phases are expressed as follow:

$$
\left\{\begin{array}{l}
\varnothing\left(f_{1}+f_{2}\right)=\emptyset\left(f_{1}\right)+\emptyset\left(f_{2}\right) \\
\varnothing\left(f_{1}-f_{2}\right)=\emptyset\left(f_{1}\right)-\emptyset\left(f_{2}\right)
\end{array}\right.
$$

The substitution of Eq. (19) into Eq. (18) presents the overall phase of the MSB is zero and its amplitude can be calculated from the product of the four magnitudes, and the maximum of the complex product will be represented. Thence, a bispectral peak will appear at bifrequency $\left(f_{1}, f_{2}\right)$. The Eq. (17) contains the $\left(f_{1}+f_{2}\right)$ and $\left(f_{1}-f_{2}\right)$ to estimate the nonlinearity of the amplitude modulation signal. If $\left(f_{1}+f_{2}\right)$ and $\left(f_{1}-f_{2}\right)$ are both due to nonlinear effect between $f_{1}$ and $f_{2}$, the bispectral peak will appear at bifrequency $B_{M S}\left(f_{1}, f_{2}\right)$. Thus, it can be concluded that the MSB can accurately and efficiently decompose the amplitude modulated signal.

To quantify the amplitude of the sideband more accurately, the MSB is modified using magnitude normalization to eliminate the amplitude contribution of carrier frequency. The MSB sideband estimator (MSB-SE) is introduced as:

$$
B_{M S}^{S E}\left(f_{1}, f_{2}\right)=\frac{B_{M S}\left(f_{1}, f_{2}\right)}{\sqrt{\left|B_{M S}\left(f_{1}, 0\right)\right|}}
$$

where $B_{M S}\left(f_{1}, 0\right)$ indicates the squared power spectrum estimation at $f_{2}=0$. Fig. 7 illustrates an example of the analysis results using MSB-SE. 


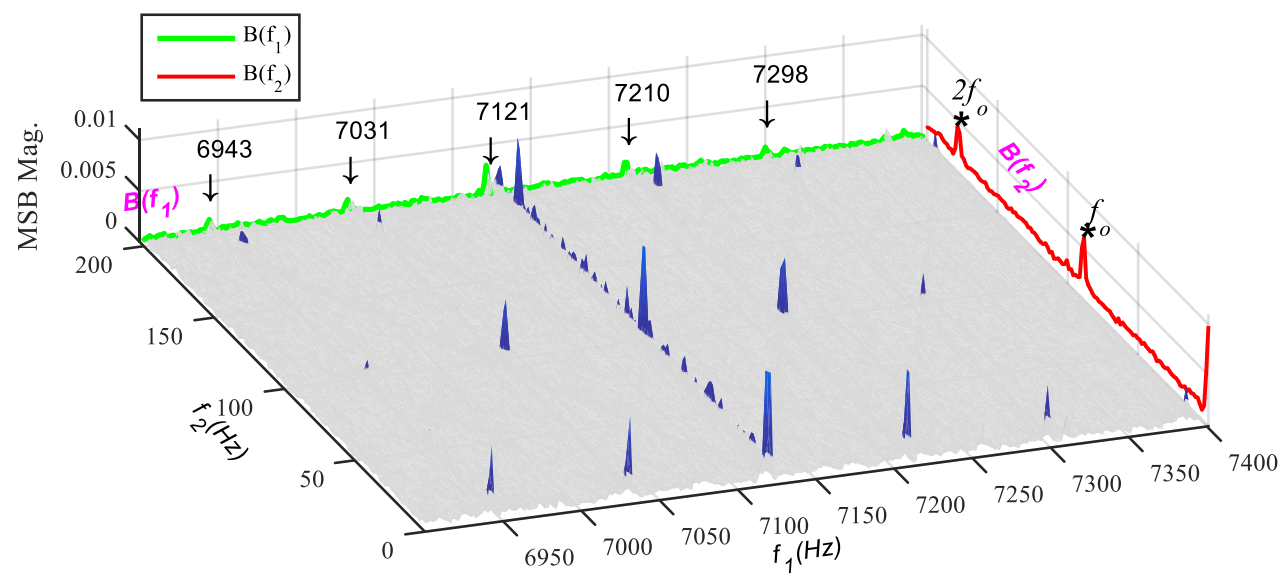

Fig. 7. Analysis results of the MSB-SE.

\section{MSB-based detector}

In order to carry out effective fault feature extraction, it is essential to select the optimal frequency band. In general, when using the MSB to analyze the measured signals, there are several suboptimal MSB slice locations with more obvious fault features. To avoid the loss of useful fault information, this paper proposes the characteristic frequency intensity coefficient (CFIC) ${ }^{51}$ to select the suboptimal MSB slices, which effectively enhances the sensitive fault components and weakens the insensitive ones. The compound MSB slice $B\left(f_{1}\right)$ is then obtained based on the average of significant MSB peaks in the incremental orientation of $f_{2}$, which is defined as:

$$
B\left(f_{1}\right)=\frac{1}{M-1} \sum_{m=2}^{M} B_{M S}^{S E}\left(f_{1}, m \Delta g\right)
$$

where $\Delta g$ indicates the frequency resolution in the $f_{2}$ direction. Based on the compound MSB slice $B\left(f_{1}\right)$ analysis, the CFIC is expressed as:

$$
\text { CFIC }=\frac{\frac{1}{M-1} \sum_{k=1}^{q} B_{M S}^{S E}\left(f_{1}, k f\right)}{\frac{1}{M-1} \sum_{m=2}^{M} B_{M S}^{S E}\left(f_{1}, m \Delta g\right)}=\frac{\sum_{k=1}^{q} B_{M S}^{S E}\left(f_{1}, k f\right)}{\sum_{m=2}^{M} B_{M S}^{S E}\left(f_{1}, m \Delta g\right)}
$$

where $B_{M S}^{S E}\left(f_{1}, k f\right)$ indicates the amplitude at the $k$ th harmonic of the defective frequencies, $k=$ $1,2, \cdots, q . f$ means the multiple of fault characteristic frequencies.

To obtain more robust results, the MSB detector is improved in accordance with the average of a few selected suboptimal slices with '*' makers in Fig. 8(a) and is defined as:

$$
B\left(f_{2}\right)=\frac{1}{N} \sum_{n=1}^{N} B_{M S}^{S E}\left(f_{1}^{n}, f_{2}\right), \quad\left(f_{2}>0\right)
$$

where $N$ stands the whole number of selected $f_{1}$ suboptimal slices (3, in the case of Fig. 8(a)), which relies on the number of CFIC. The MSB detector is depicted in Fig. 8(b). 

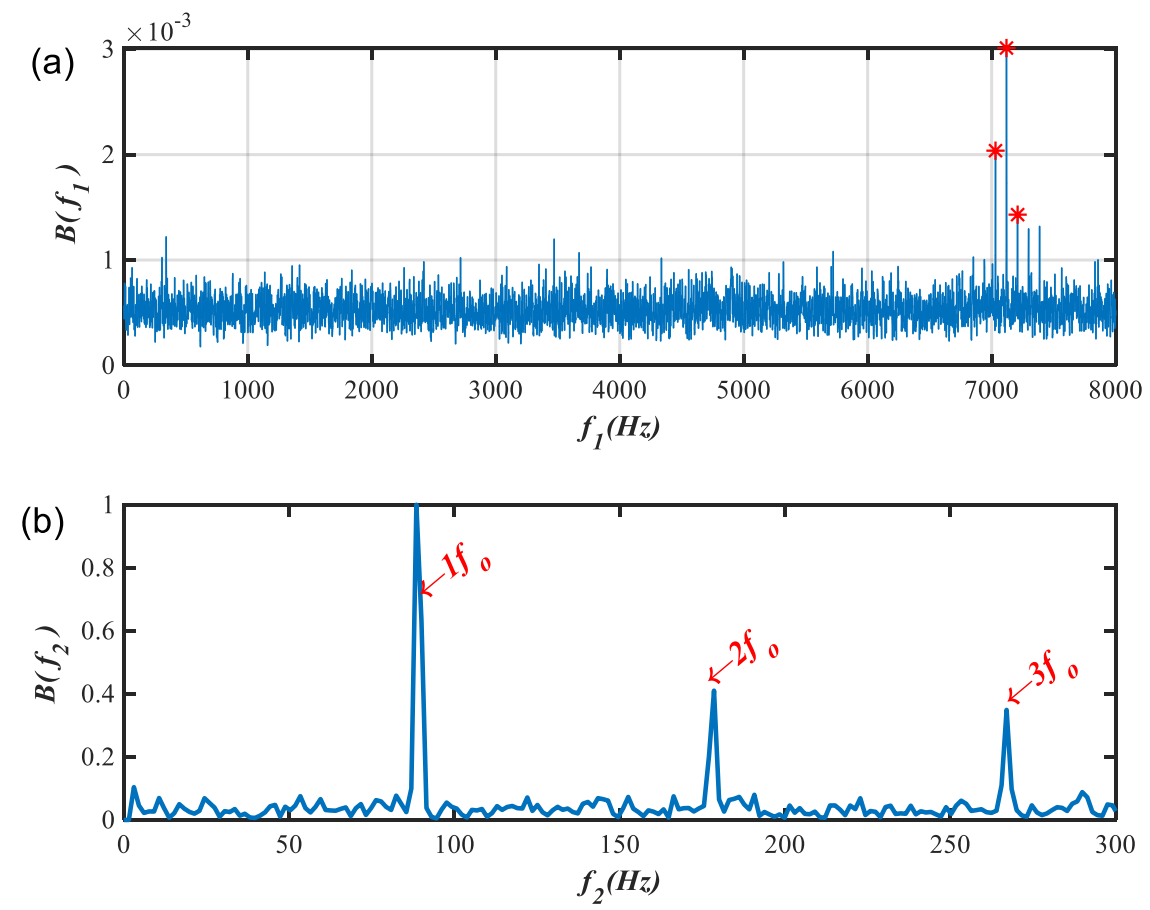

Fig. 8. Analysis results of MSB: (a) MSB slice $B\left(f_{1}\right)$; (b) MSB detector.

\section{The proposed fault diagnosis scheme}

In this section, the proposed fault detection scheme based on WPD and MSB is introduced in detail. The WPD successfully makes use of the transient impulse enhancement to remove most of the interference contents while preserving the fault signature information. The MSB is then used to further denoise the signal and extract the characteristic frequencies associated with the fault based on the modulation component demodulation. The proposed fault diagnosis scheme is described as follow:

Step 1: Implement binary WPT on the measured signal to obtain a series of time-frequency subspaces.

Step 2: Optimize the threshold value of wavelet threshold denoising according to Fig. 1.

Step 3: Select the optimal threshold value for applying WPD on the time-frequency subspaces.

Step 4: Calculate the NWPE matrix on the denoising subspaces and select the master subspaces using the GI to obtain transient impulses.

Step 5: Perform the MSB on the transient impulses and then select suboptimal MSB slices based on CFIC criteria.

Step 6: Obtain the MSB detector by averaging the selected suboptimal MSB slices and applying them to infer the type of bearing fault.

\section{Simulation analysis}

To show the performance of the proposed WPD-MSB method, the simulated signal with $2 \%$ slippage is taken as the baseline signal but added to higher level of random noise. Fig. 9(a) shows both the baseline signal and its noise added one $(\mathrm{SNR}=-20 \mathrm{~dB})$. It can be seen that the impulsive feature information is completely overwhelmed by the noise. In the frequency domain as illustrated in Fig. 9(b), only slight higher spectral lines can be observed in the resonance bands around $7 \mathrm{kHz}$. It is very 
challenging to extract the information to reflect the impulsive feature.
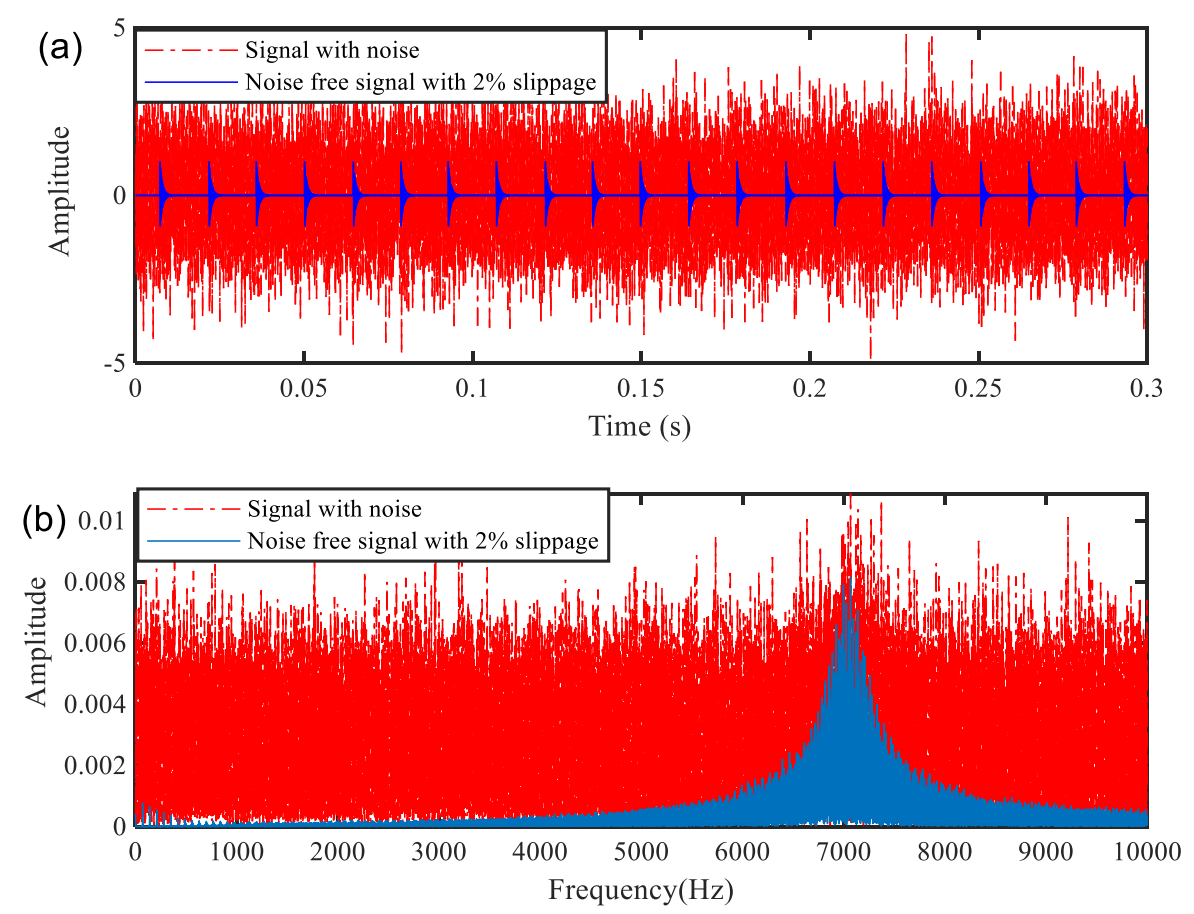

Fig. 9. Simulated signal of an outer race defect with the SNR of $-20 \mathrm{~dB}$ : (a) waveform with $2 \%$ random slippage; (b) spectrum of the (a).

To obtain the impulsive fault information, WPD-MSB method was applied to the simulated signal. Firstly, the simulated signal depicted in Fig. 9(a) was decomposed into 32 time-frequency subspaces using the Meyer wavelet. The time-frequency subspaces were then denoised by the WPD, an optimal threshold value $\Delta_{m}^{o}=1.1552$ was determined as shown in Fig. 10. This optimal value is much higher compared with that case when $\mathrm{SNR}=-10 \mathrm{~dB}$ as displayed in Fig. 4, showing that a higher threshold is needed for high noise contamination. Furthermore, NWPE matrix of denoising subspaces was also calculated, and these matrices were made into a list of histograms as displayed in Fig. 11. It found that the NWPE values at node [5,4] (band is $6000 \sim 7500 \mathrm{~Hz}$ ) show significantly high amplitude, allowing an optimal band to be selected for further analysis using MSB. Finally, the denoised signal was reconstructed as illustrated in Fig. 12. It can be seen that the impulsive features in the waveform are greatly enhanced. However, there still exists clear randomness, making it difficult to see the deterministic periodicity for fault detection. 


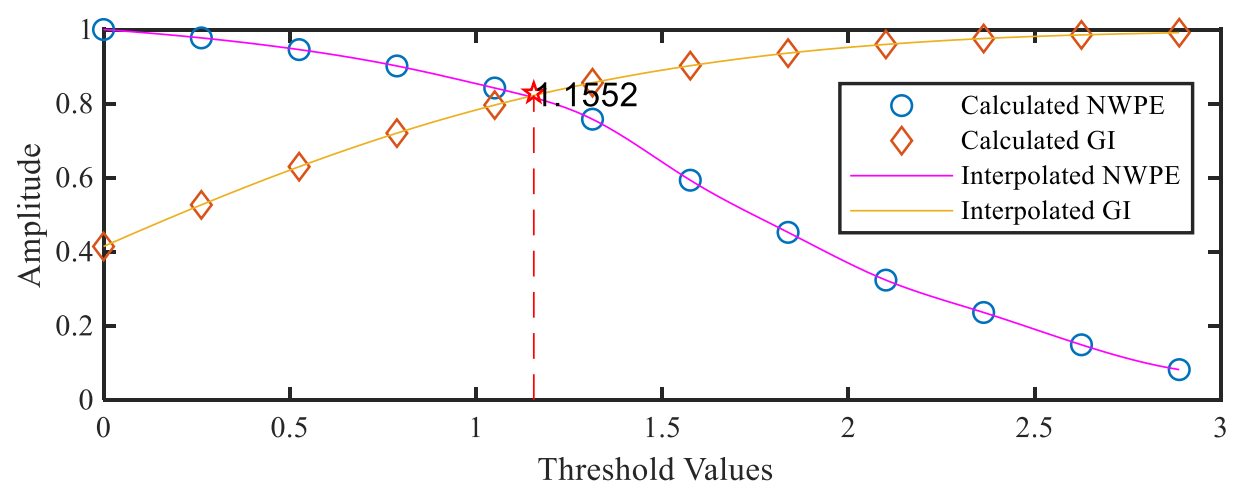

Fig. 10. Result of the threshold value optimization.

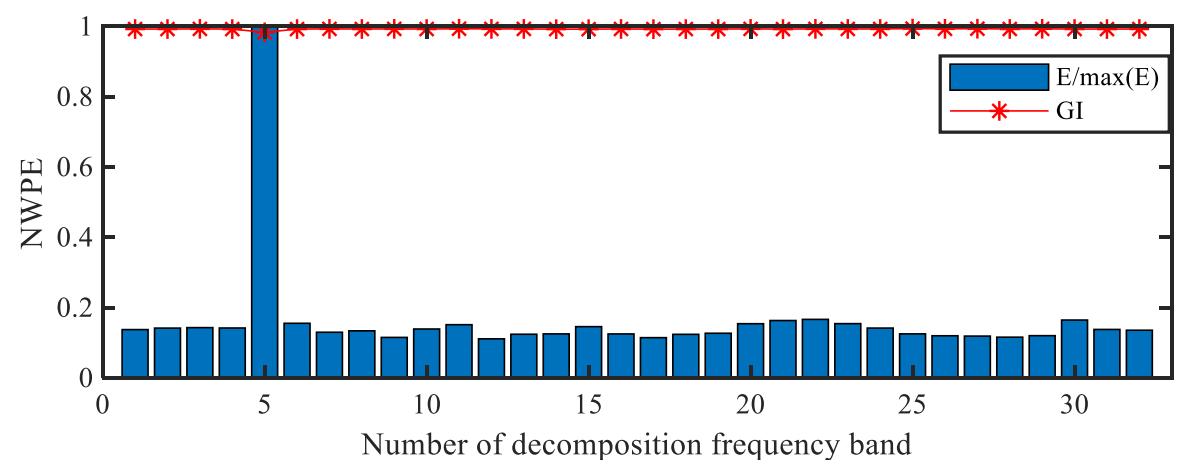

Fig. 11. Proportional histogram of the NWPE distribution.

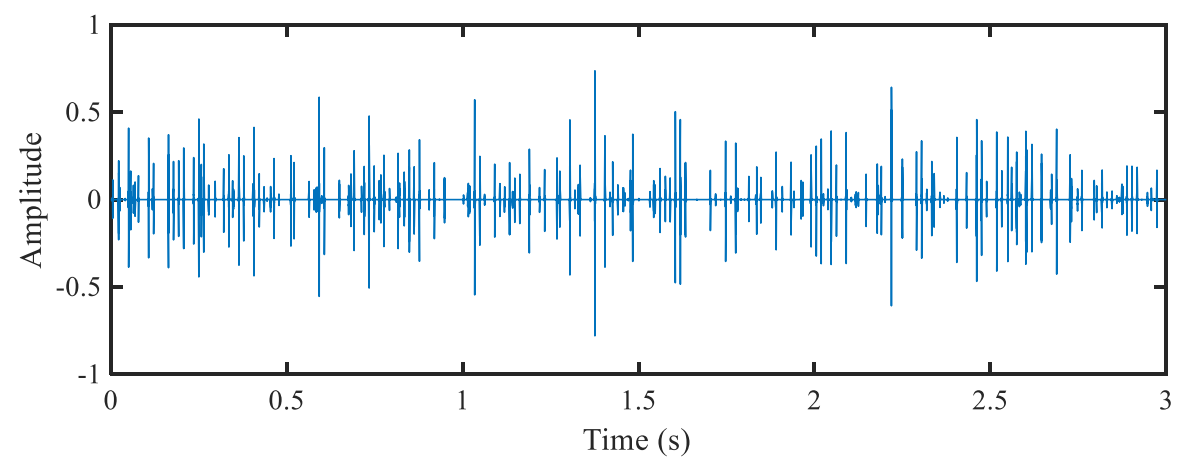

Fig. 12. Waveform of the reconstructed signal.

To find the frequency characteristic in the reconstructed signal, MSB and MSB-SE were computed using Eqs. (17) and (20). The MSB slice $B\left(f_{1}\right)$ was obtained using Eq. (21) and the suboptimal MSB slices were selected based on the proposed CFIC criteria. The number of the suboptimal MSB slices occurs at 3 MSB slices with the maximum CFIC value in Fig. 13. Moreover, it is worth noting that the suboptimal MSB slices are sorted from max $\left(B\left(f_{1}\right)\right)$ to $\min \left(B\left(f_{1}\right)\right)$. Subsequently, the MSB detector can be calculated by averaging the selected 3 suboptimal MSB slices, and the detection result is obtained and presented in Fig. 14. It shows distinctive peaks corresponding exactly to the outer race fault frequency $f_{o}$ and its harmonics. 


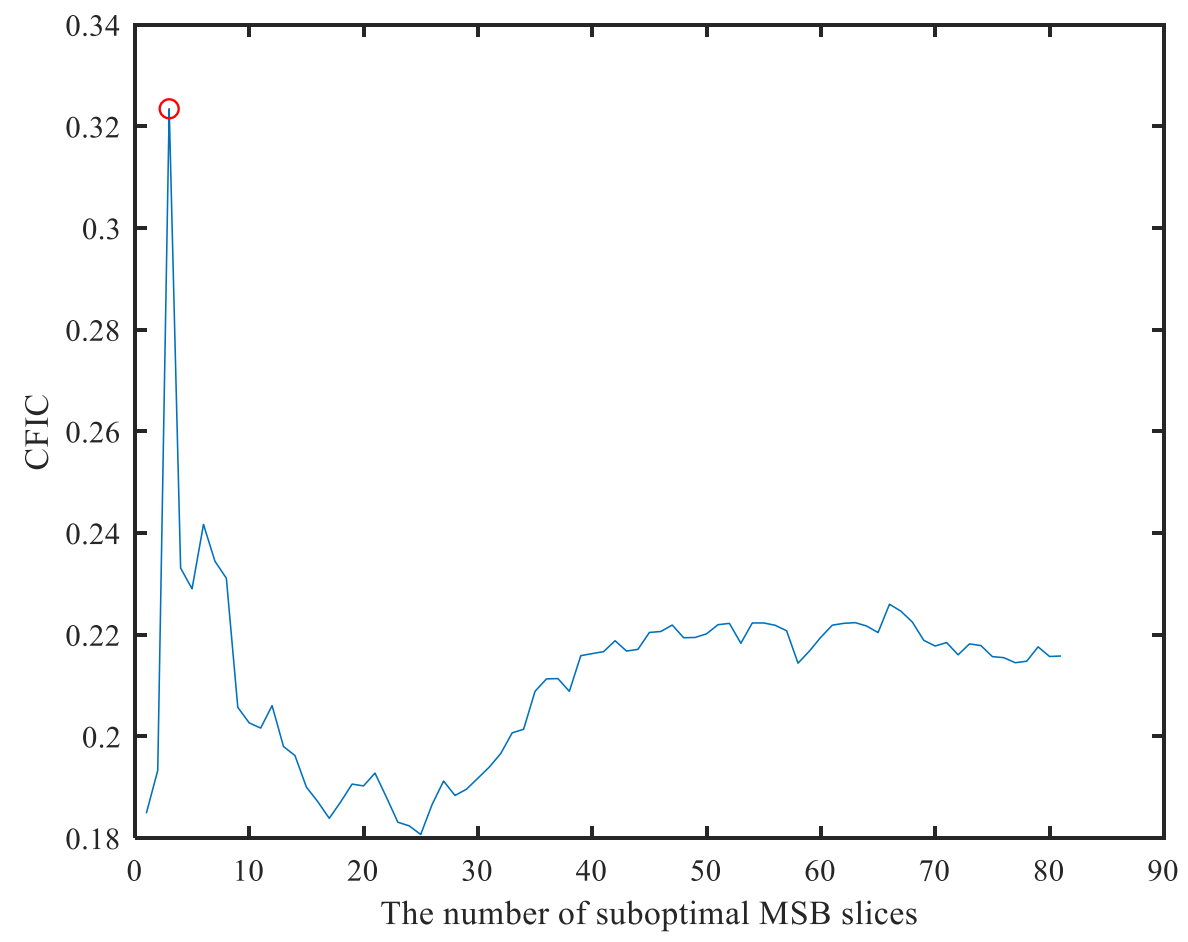

Fig. 13. CFIC value of suboptimal MSB slices.

For comparison, the conventional MSB in Ref. [35] was utilized to process simulated signals that are not denoised by WPD. Fig. 15 presents the fault features and detection results using the conventional MSB. Although the analysis result can reflect the outer race fault frequencies $f_{o}$ and its harmonics, the interference components are much higher than the analysis result depicted in the Fig. 14. It proved that the WPD-MSB is more capable of extracting the fault characteristic frequencies under strong background noise effectively.
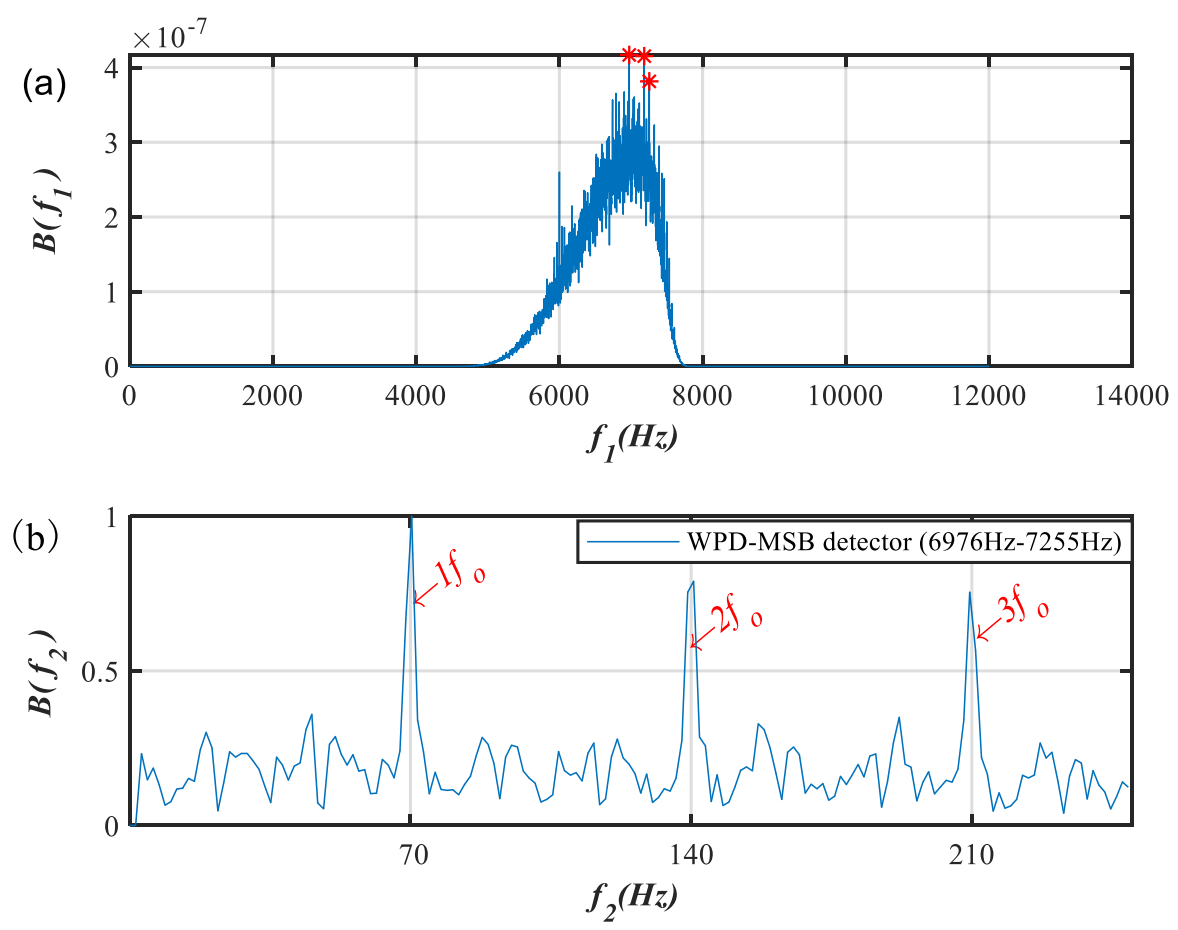
Fig. 14. Results of WPD-MSB: (a) WPD-MSB slices; (b) WPD-MSB detector.
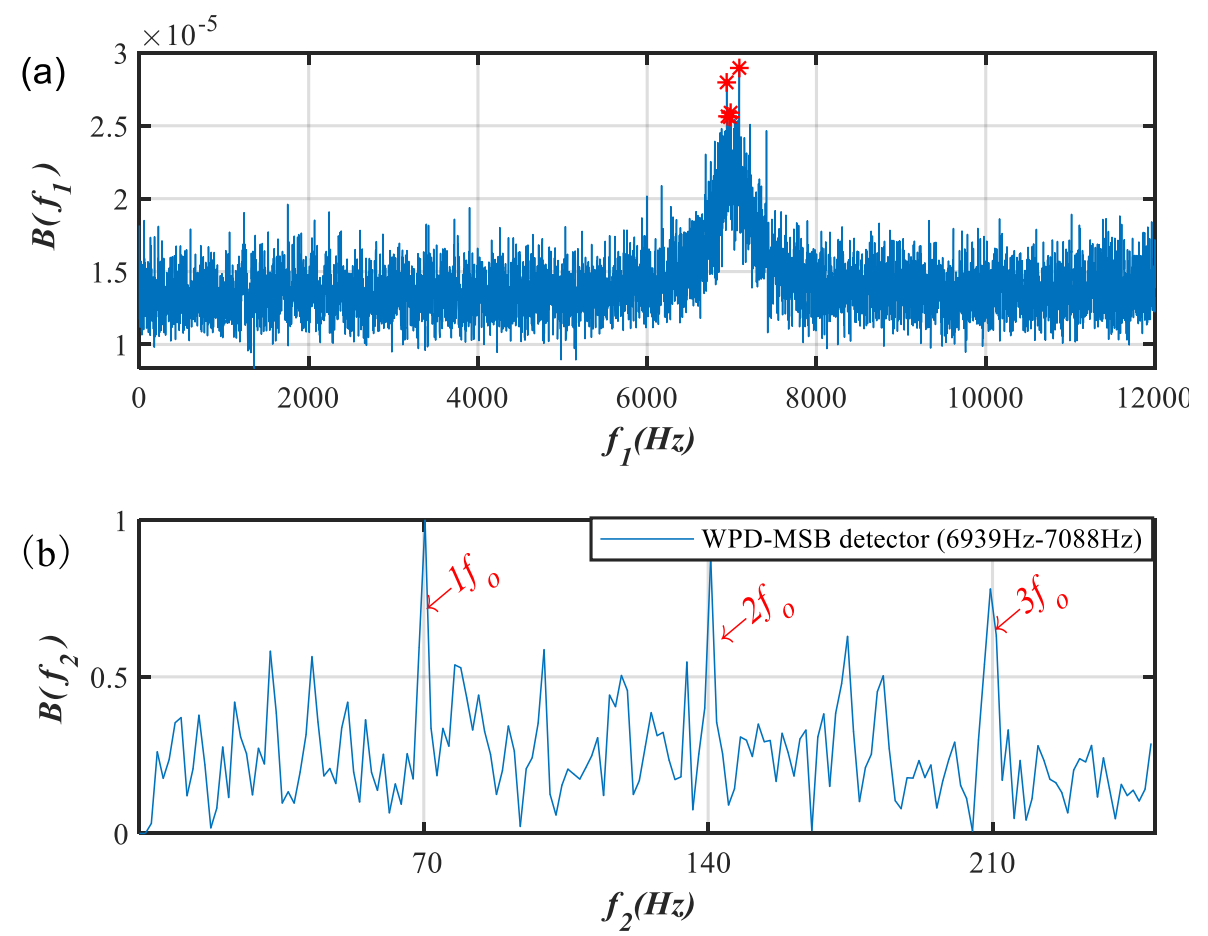

Fig. 15. Results of the conventional MSB: (a) MSB slices; (b) MSB detector.

\section{Experiment validation}

In this section, two experimental case studies are conducted to validate the performance and effectiveness the WPD-MSB for bearing fault detection. Additionally, the validity of the WPD-MSB was compared with the variational mode decomposition and Teager energy operator (VMD-TEO), fast kurtogram (FK) and the conventional MSB in the experimental case analysis.

\section{Motor bearing outer race fault detection}

\section{Test setup and data collection}

The first experimental case is the fault detection for the outer race of a motor bearing. The experimental data were obtained from the bearing test platform as depicted in Fig. 16. The experimental device comprises an AC motor (right), dynamic brake (left), two supporting bearings and three flexible coupling as well as an accelerometer sensor. The accelerometer sensor was mounted in the vertical direction of the motor bearing housing. Fig. 17 illustrates the motor bearing outer race with small defect. The sampling frequency for collecting the experiment data was $96 \mathrm{kHz}$ and the data length were 1920,000 points. The specifications and fault defect frequencies of the motor bearing were given in Table 2 . 


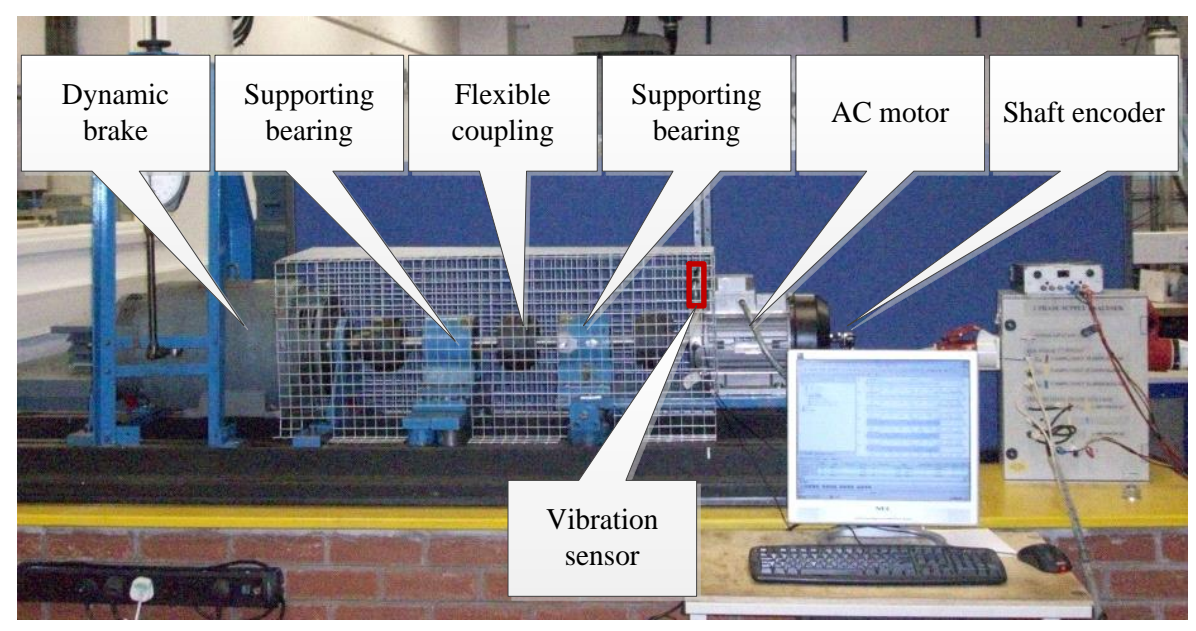

Fig. 16. The test platform of the motor bearing.
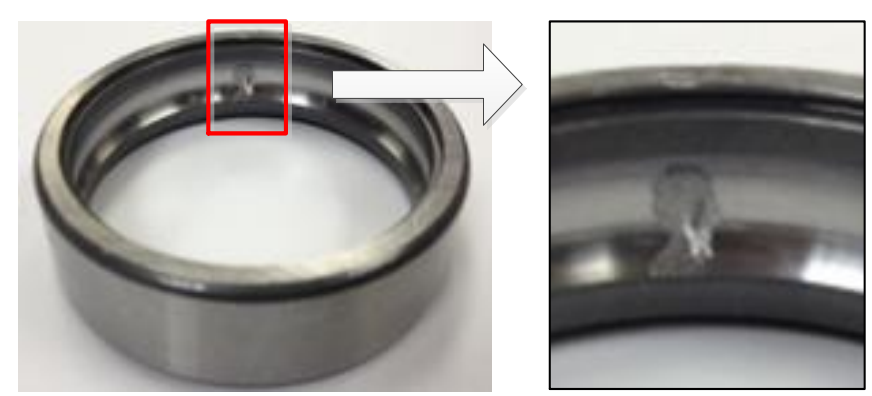

Fig. 17. The motor bearing outer race with small defect.

Table 2. Main specifications and fault defect frequencies of the motor bearing.

\begin{tabular}{|c|c|c|c|c|}
\hline \multirow{2}{*}{$\begin{array}{c}\text { Bearing } \\
\text { designation }\end{array}$} & $\begin{array}{c}\text { Ball diameter } \\
d(\mathrm{~mm}) \\
\end{array}$ & $\begin{array}{c}\text { Pitch diameter } \\
\qquad D_{m}(\mathrm{~mm})\end{array}$ & $\begin{array}{c}\text { Ball number } \\
z \\
\end{array}$ & $\begin{array}{c}\text { Contact angle } \\
\beta \\
\end{array}$ \\
\hline & 9.53 & 46.4 & 9 & $0^{\circ}$ \\
\hline \multirow{2}{*}{$6206 Z Z$} & $f_{o}$ & $f_{i}$ & $f_{b}$ & $f_{c}$ \\
\hline & 89.39 & 130.99 & 62.42 & 9.93 \\
\hline
\end{tabular}

\section{Results and analysis}

Fig. 18 depicts the waveform of the measured faulty bearing signal and its corresponding spectrum. Although the fault defect frequencies (i.e. $2 f_{o}$ and $3 f_{o}$ ) can be identified, there are still interference frequencies as shown in Fig. 18(b). Therefore, it is highly significant to remove these interference components to obtain the fault defect frequencies effectively. 

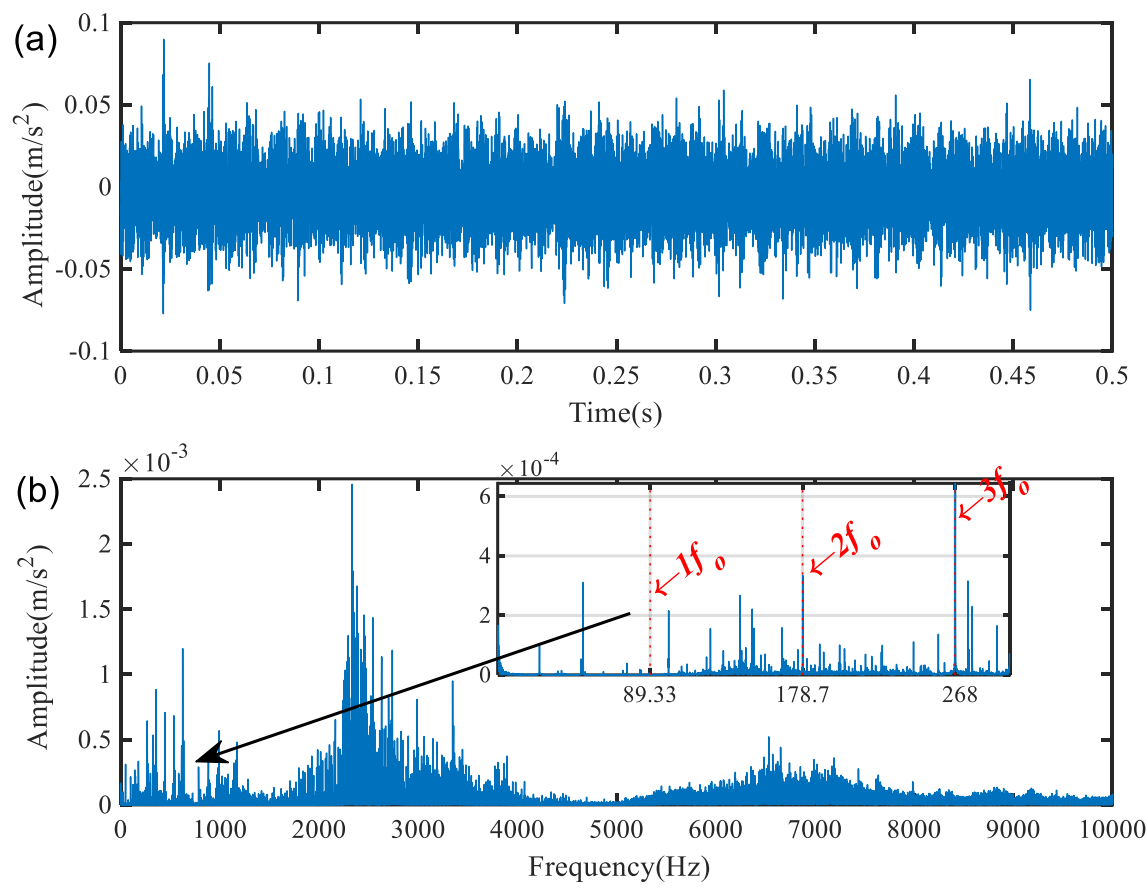

Fig. 18. Raw date of the faulty bearing in motor: (a) waveform; (b) spectrum.

The proposed WPD-MSB method was then utilized to process the measured signal of the faulty bearing. The faulty bearing signal displayed in Fig. 18(a) was decomposed into 32 time-frequency subspaces using the Meyer wavelet, and the threshold criterion $F_{m}$ was then utilized to determine the optimal threshold value $\Delta_{m}^{o}$ for WPD application. The result of the threshold value optimization was shown in Fig. 19. Obviously, an optimal threshold value $\Delta_{m}^{o}=0.0139$ can be selected according to the analysis results illustrated in Fig. 19. Hereafter, the NWPE matrix of denoising subspaces was calculated, and the proportional histogram of the NWPE distribution of the motor bearing outer race fault was depicted in Fig. 20. It found that the NWPE at node nodes [5,0], [5,5] and [5,6] can be selected as the master subspaces. Finally, the denoised signal was reconstructed and illustrated in Fig. 21. Although the impulsive features in the waveform are enhanced, there is still obvious randomness, so it is difficult to see the deterministic periodicity for fault detection.

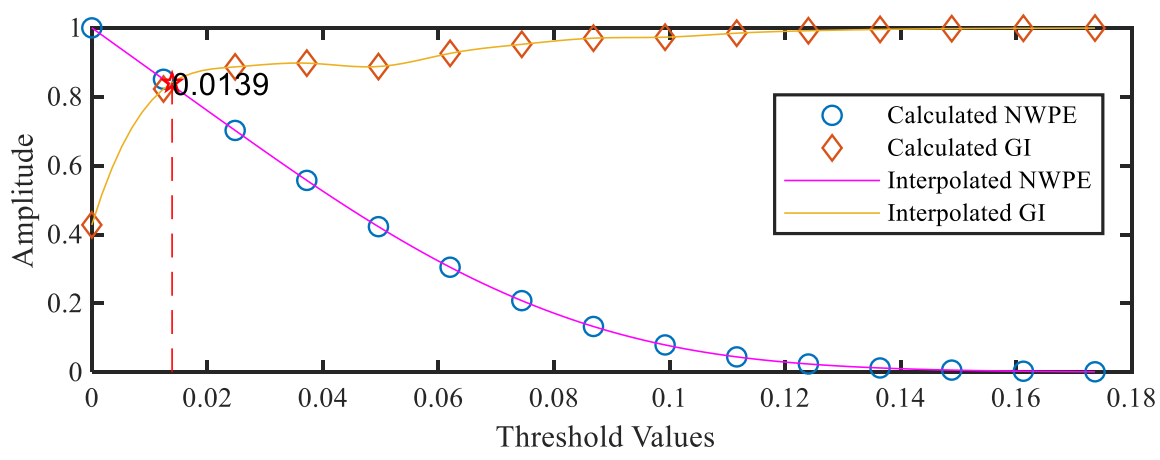

Fig. 19. Result of the threshold value optimization. 


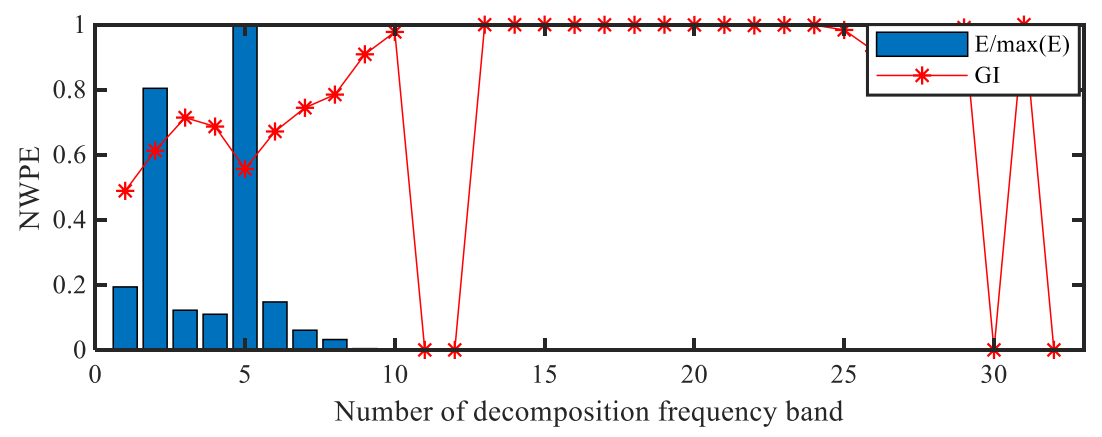

Fig. 20. Proportional histogram of the NWPE distribution.

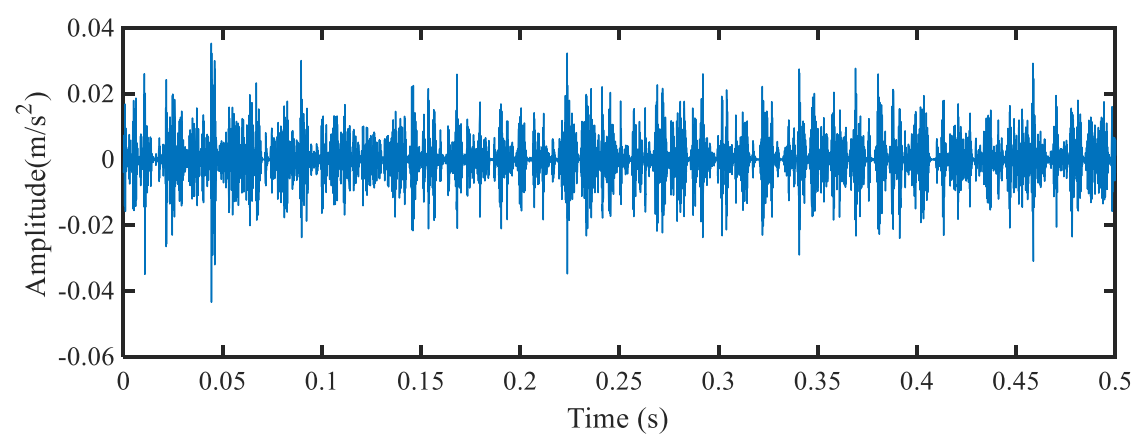

Fig. 21. Waveform of the reconstructed signal.

The envelope analysis was then used to analyze the reconstructed signal to extract the fault defect frequencies as shown in Fig. 22. The results can present the outer race fault frequencies $f_{o}$ and its harmonics, but the noise interference is much high and the modulation effects of the bearing fault are not obvious, especially around the higher harmonics. Subsequently, the MSB was exploited to analyze the reconstructed signal instead of performing envelope analysis to obtain the fault defect frequencies. The number of suboptimal MSB slices occurs at 3 with the maximum CFIC value as illustrated in Fig. 23. The MSB detector was computed by averaging the 3 suboptimal MSB slices, and the detection result was depicted in Fig. 24. It can be found that the WPD-MSB has distinctive peaks corresponding to the outer race fault frequency $f_{o}$ and its harmonics. This indicates that the defects on the motor bearing are effectively evaluated by employing the WPD-MSB. In addition, the cage fault frequency $f_{\text {cage }}$ and its harmonics are also effectively extracted. The existence of these peaks may be due to manufacturing accuracy or improper installation.

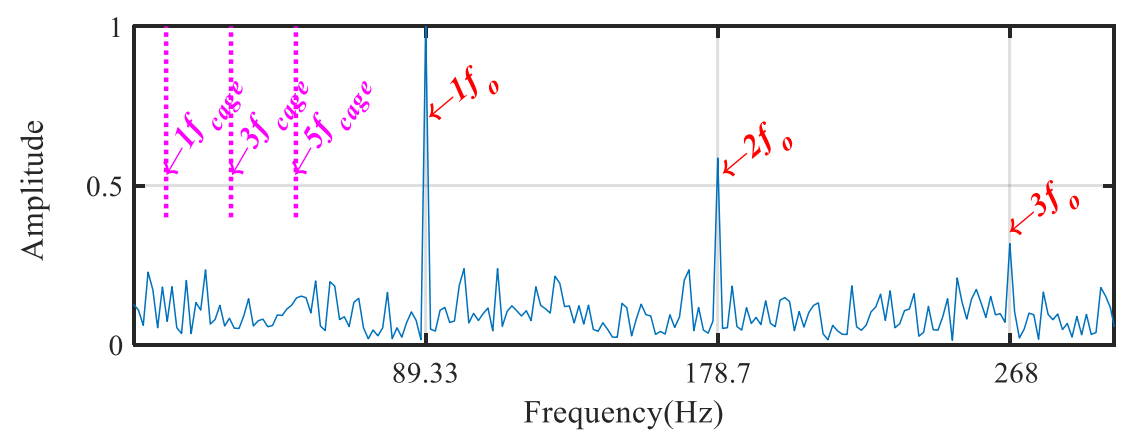


Fig. 22. Envelope spectrum of the reconstructed signal.

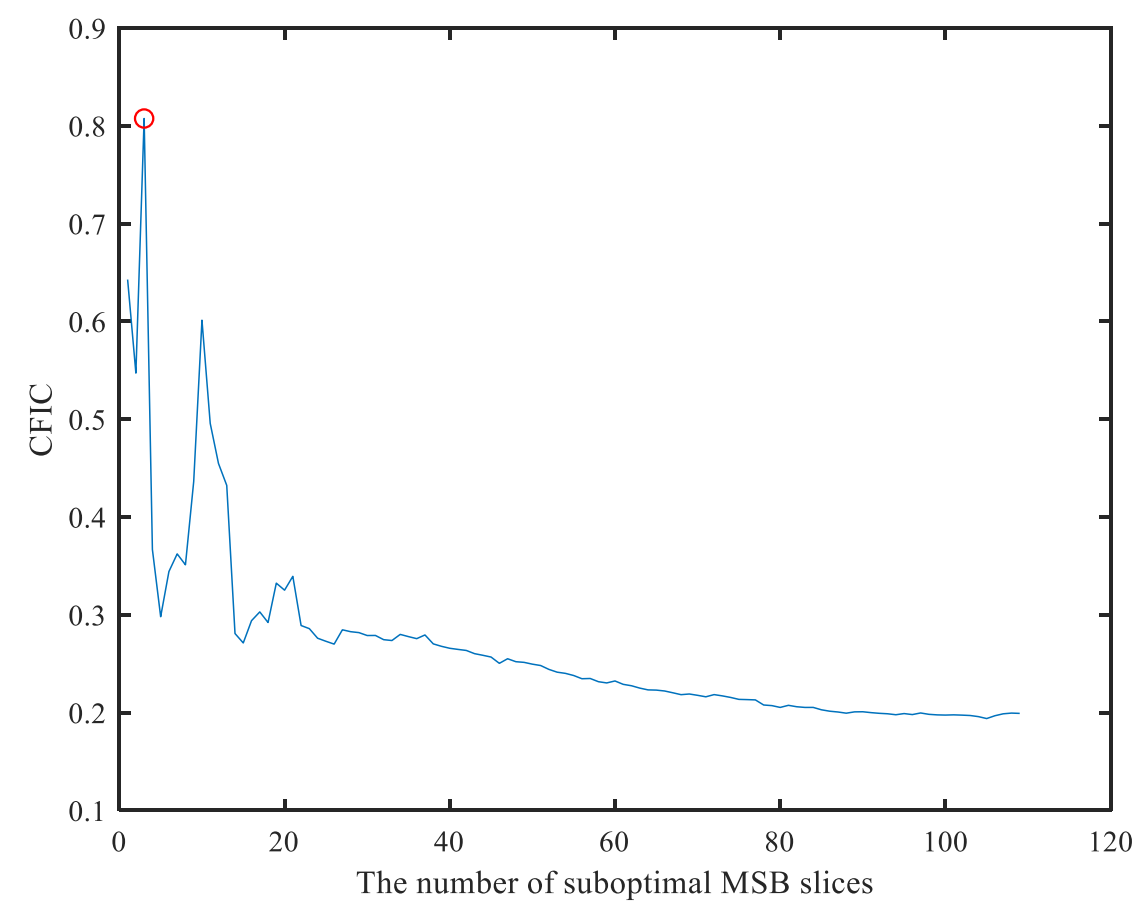

Fig. 23. CFIC value of suboptimal MSB slices.
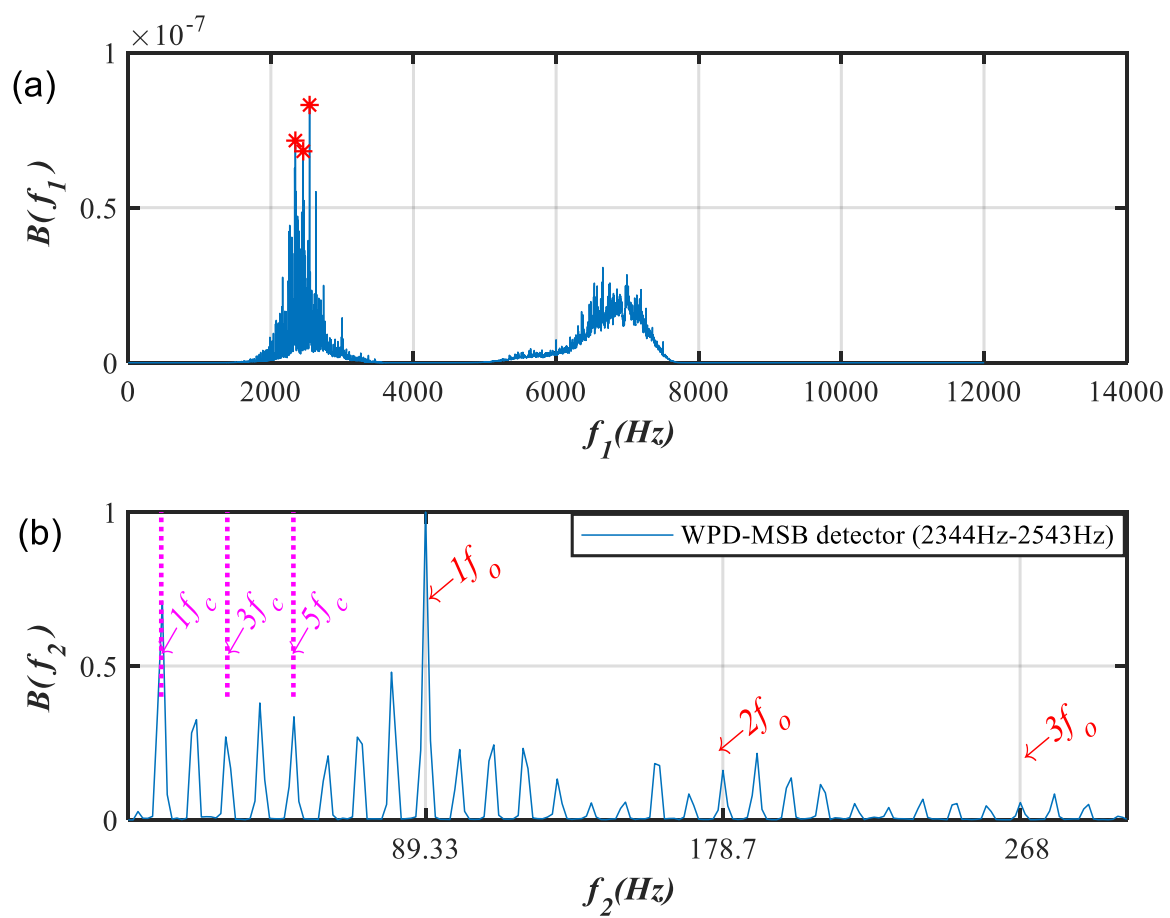

Fig. 24. Results of WPD-MSB: (a) WPD-MSB slices; (b) WPD-MSB detector.

As a comparison, the VMD-TEO ${ }^{52}$ and fast kurtogram $(\mathrm{FK})^{53}$ were applied to analyze the same experimental data. Initially, the inside parameters $\alpha$ and decomposition number $K$ were set as 200 and 6, respectively. The decomposition results of the VMD and its spectrum were presented in Fig. 25. Compared with other IMFs, the frequency band of IMF1 was consistent with the main resonance 
frequency band of the motor bearing fault signal. Subsequently, the Teager energy operator (TEO) was employed on the IMF1 to obtain fault defect frequencies, and the result was depicted in Fig. 26. As depicted in the VMD-TEO spectrum, the outer race fault frequencies (i.e. $f_{o}, 2 f_{o}$ and $3 f_{o}$ ) are visible, but there are interference frequencies around the higher-frequency harmonics. Moreover, the cage fault frequency $f_{\text {cage }}$ and its harmonics cannot be accurately obtained. Similarly, the results of the FK are illustrated in Fig. 27. The bandwidth and central frequency of the band pass filter are $375 \mathrm{~Hz}$ and 26438 $\mathrm{Hz}$ as depicted in Fig. 27(a). The envelope and squared envelope spectrum of the filtered signal by FK are illustrated in Fig. 27(b) and (c). From Fig. 27(c), the cage frequencies $f_{\text {cage }}$ and its harmonics, the outer race fault frequencies $f_{o}$ and its harmonics can be recognized, but there are still a lot of noisy signals and interference components.
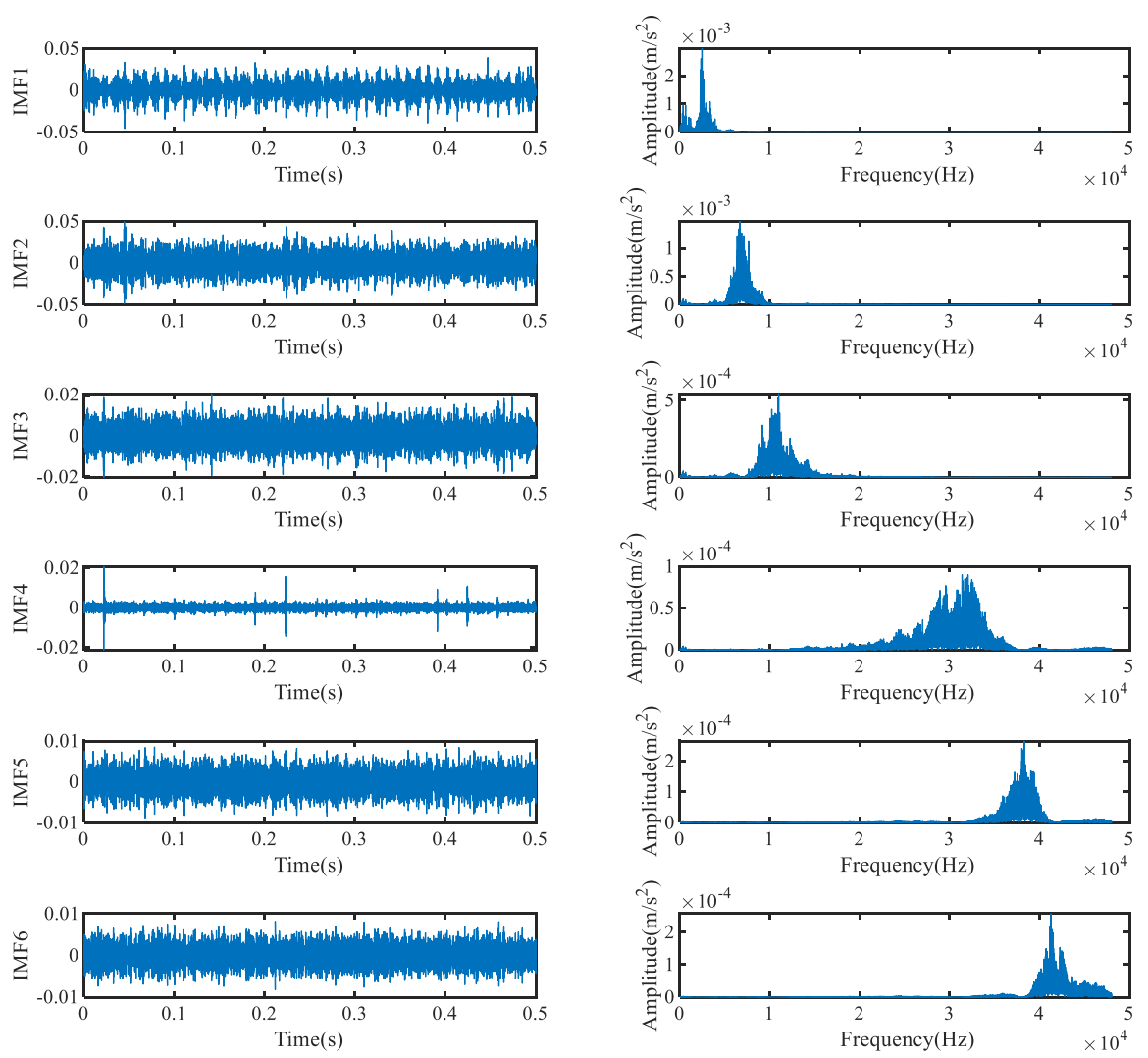

Fig. 25. Analysis results of the motor bearing signal by VMD: (a) waveform; (b) spectrum. 

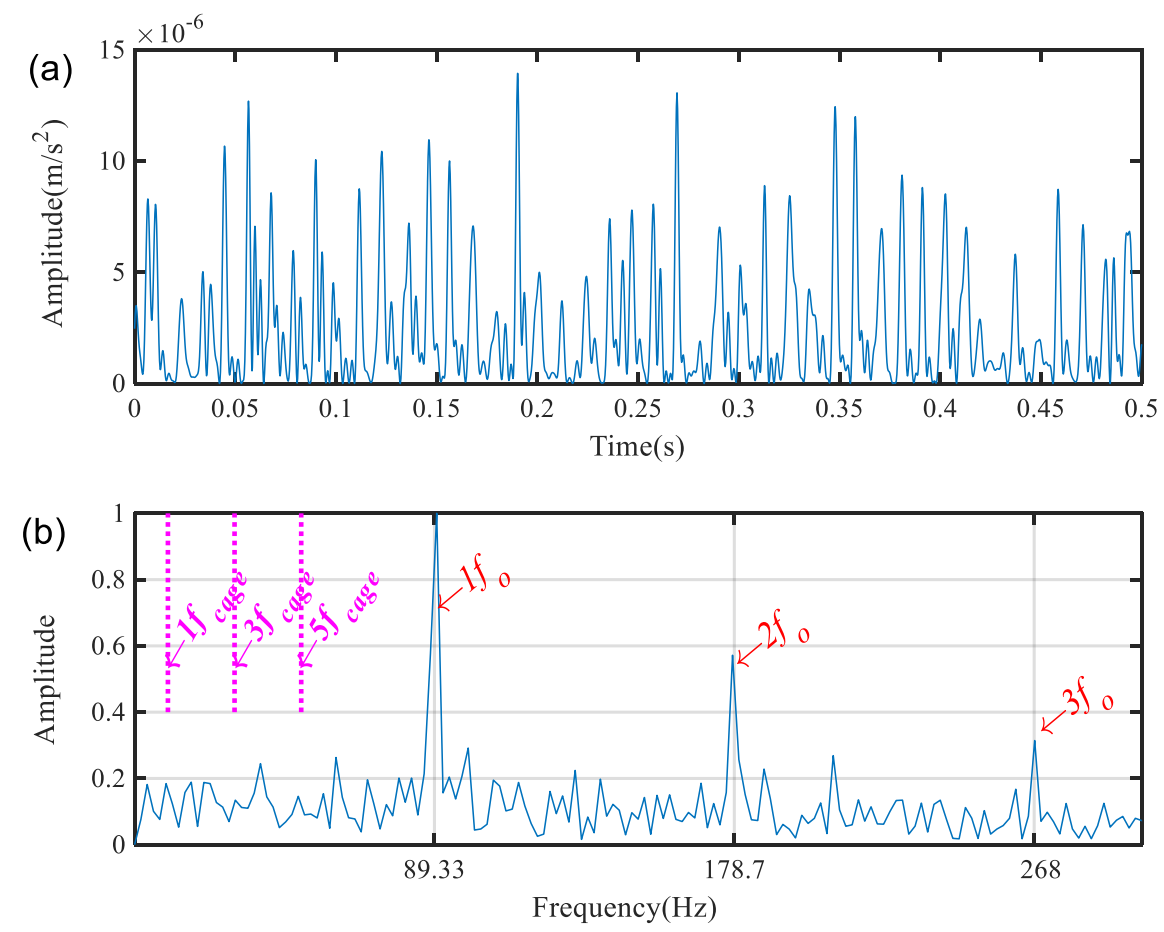

Fig. 26. Results of VMD-TEO: (a) waveform; (b) spectrum with central frequency of $2400 \mathrm{~Hz}$ and a bandwidth of $600 \mathrm{~Hz}$.
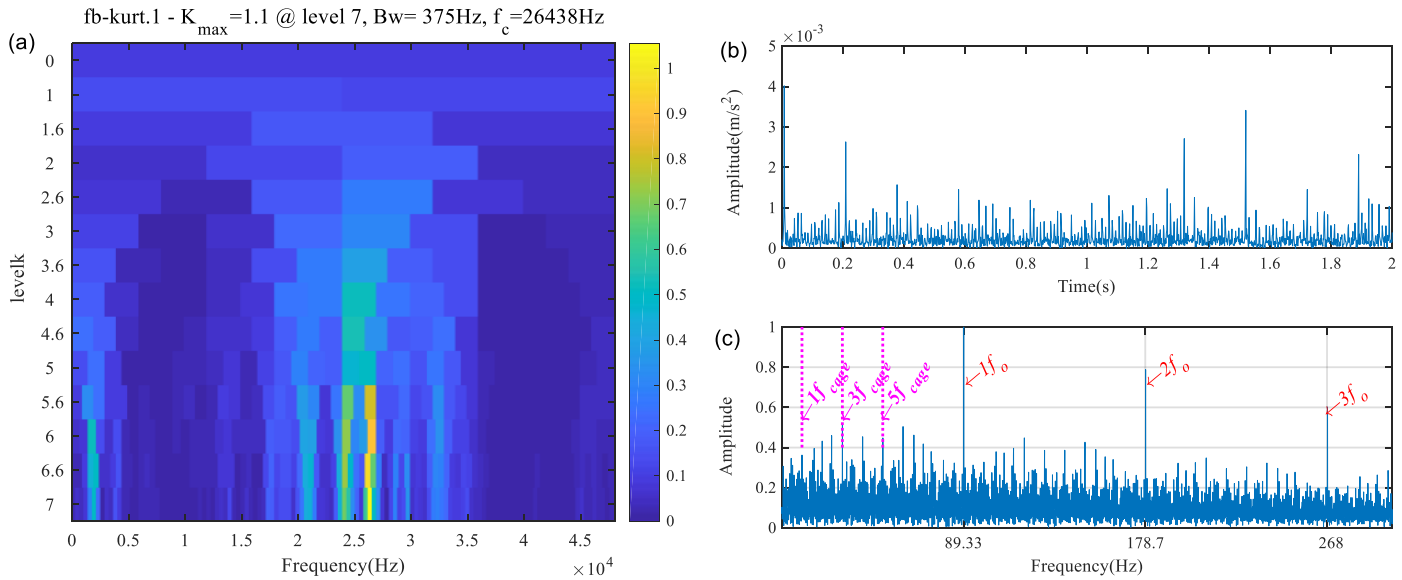

Fig. 27. Results of FK: (a) kurtogram; (b) envelope; (c) squared envelope spectrum.

To further prove the superiority of the WPD-MSB algorithm, the conventional MSB was utilized to process vibration signals and the detection results were depicted in Fig. 28. As can be seen, the analysis result obtained by the conventional MSB can reflect the cage frequencies $f_{\text {cage }}$ and its harmonics, the outer race fault frequencies $f_{o}$ and its harmonics, but the interference components are much higher than the result displayed in the Fig. 24. To accurately analyze the results of the above four algorithms, a fault defect index ${ }^{54}$ was employed to evaluate their performance in extracting fault features. For a given fault defect frequency $f$, the fault defect index $\bar{\alpha}$ can be defined as follows:

$$
\bar{\alpha}=\frac{1}{N} \sum_{n=1}^{N} \frac{A(n f)}{\operatorname{mean}(A(n f-f), A(n f+f))}
$$


where $A(n f)$ indicates the magnitude at the $n$th harmonic of the fault defect frequencies, $N$ denotes the multiple of fault defect frequencies. Table 3 illustrates the fault defect index of the four algorithms. Compared with VMD-TEO, FK and MSB, the proposed WPD-MSB method has more powerful fault diagnosis capabilities. Thus, it is reasonable to reveal that the WPD-MSB can extract the motor bearing fault defect frequencies more effectively than the VMD-TEO, FK and conventional MSB method.

Table 3. Fault defect index of the four algorithms.

\begin{tabular}{cc}
\hline Methods & Values \\
\hline WPD-MSB & 7.288 \\
VMD-TEO & 5.690 \\
FK & 1.596 \\
MSB & 1.534 \\
\hline
\end{tabular}
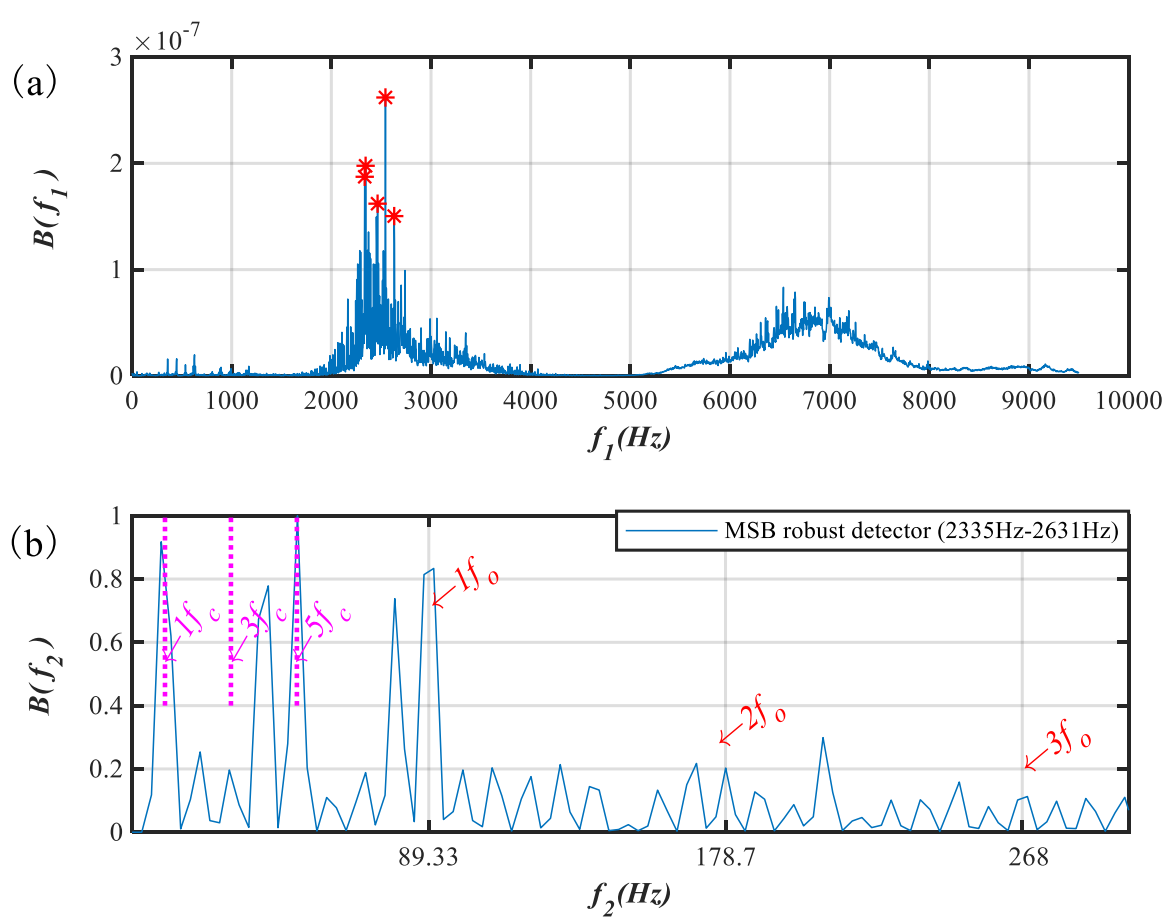

Fig. 28. Results of the conventional MSB: (a) MSB slices; (b) MSB detector.

\section{Planetary gearbox bearing inner race fault detection}

\section{Test setup and data collection}

The second experimental case is the fault detection for the inner race of a planetary gearbox bearing. The test platform is depicted in Fig. 29. The experimental device is composed of a motor (left), a planetary gearbox, two flexible type couplings, a helical gearbox, and a DC generator (right). The measured signal of the planetary gearbox was acquired using an accelerometer sensor installed on the top of the planetary gearbox with the sensitivity of $28.7 \mathrm{mV} / \mathrm{ms}^{-2}$. Fig. 30 shows the installation position of the test bearing. The sampling frequency for the experiment data collection was $96 \mathrm{kHz}$ and 
the data length were 2880,000 points. Fig. 31 illustrates the planetary gearbox bearing inner race with small defect. The specifications and fault defect frequencies of the planetary gearbox bearing were listed in Table 4.

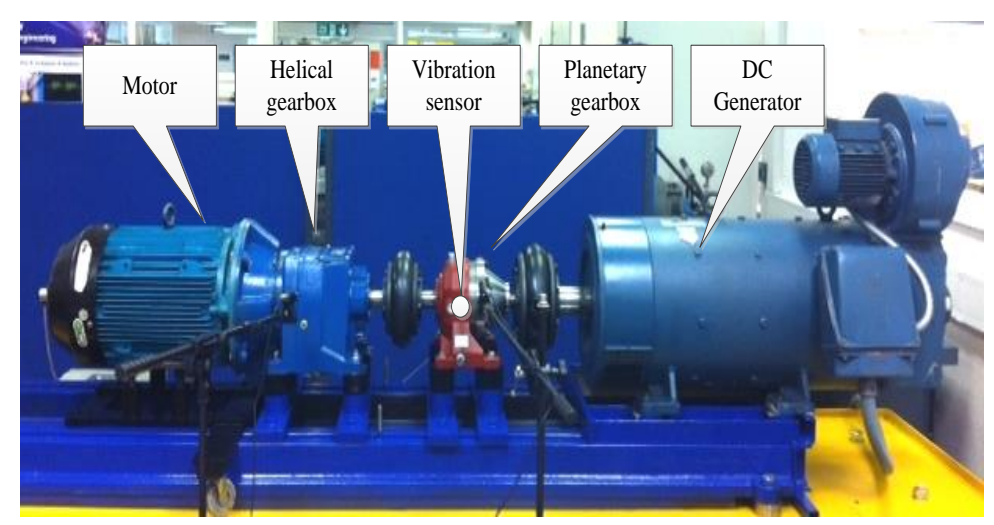

Fig. 29. The test platform of the planetary gearbox.

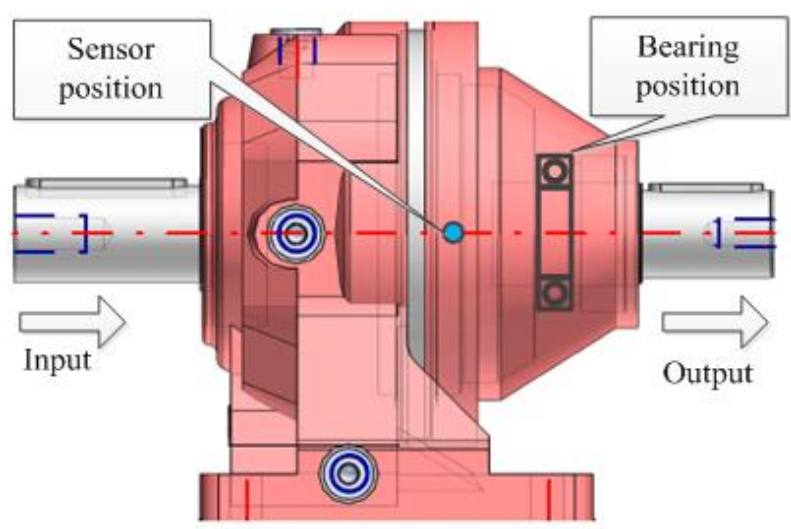

Fig. 30. The installation position of the test bearing.

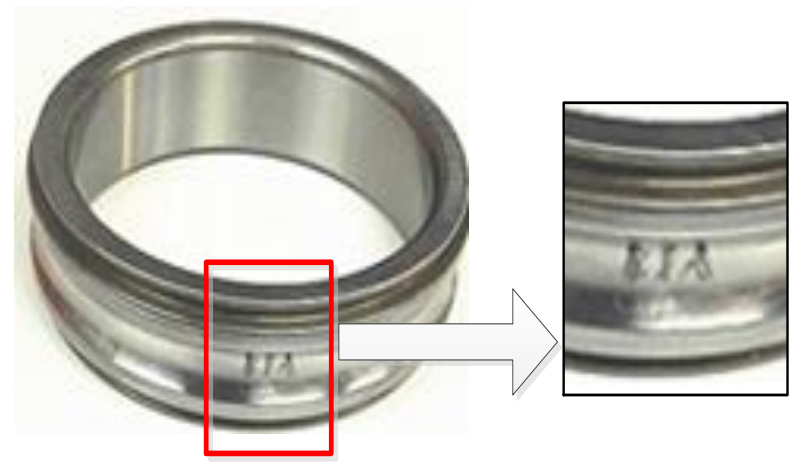

Fig. 31. The planetary gearbox bearing inner race with small defect.

Table 4. Main specifications and fault defect frequencies of the planetary gearbox bearing.

\begin{tabular}{|c|c|c|c|c|c|c|}
\hline \multirow{2}{*}{$\begin{array}{c}\text { Bearing } \\
\text { designation }\end{array}$} & \multicolumn{2}{|c|}{$\begin{array}{c}\text { Ball diameter } \\
\quad d(\mathrm{~mm}) \\
\end{array}$} & $\begin{array}{l}\text { Pitch diameter } \\
\qquad D_{m}(\mathrm{~mm})\end{array}$ & $\begin{array}{c}\text { Ball number } \\
z \\
\end{array}$ & \multicolumn{2}{|c|}{$\begin{array}{c}\text { Contact angle } \\
\beta \\
\end{array}$} \\
\hline & \multicolumn{2}{|c|}{8} & 57.5 & \multirow[t]{2}{*}{12} & \multicolumn{2}{|c|}{$0^{\circ}$} \\
\hline \multirow{2}{*}{6008} & $f_{r s}$ & $f_{s f}$ & $f_{o}$ & & $f_{b}$ & $f_{c}$ \\
\hline & 9.53 & 24.63 & 49.25 & 65.17 & 33.60 & 4.10 \\
\hline
\end{tabular}


The waveform and its spectrum of the measured vibration signal of the test planetary gearbox bearing with inner race fault are depicted in Fig. 32. Obviously, some useful fault features information is absolutely destroyed by the strong background noise as illustrated in Fig. 32(a). Besides, the interference components are plentiful, so it is extremely difficult to obtain fault defect frequency $f_{i}$ and its harmonic.
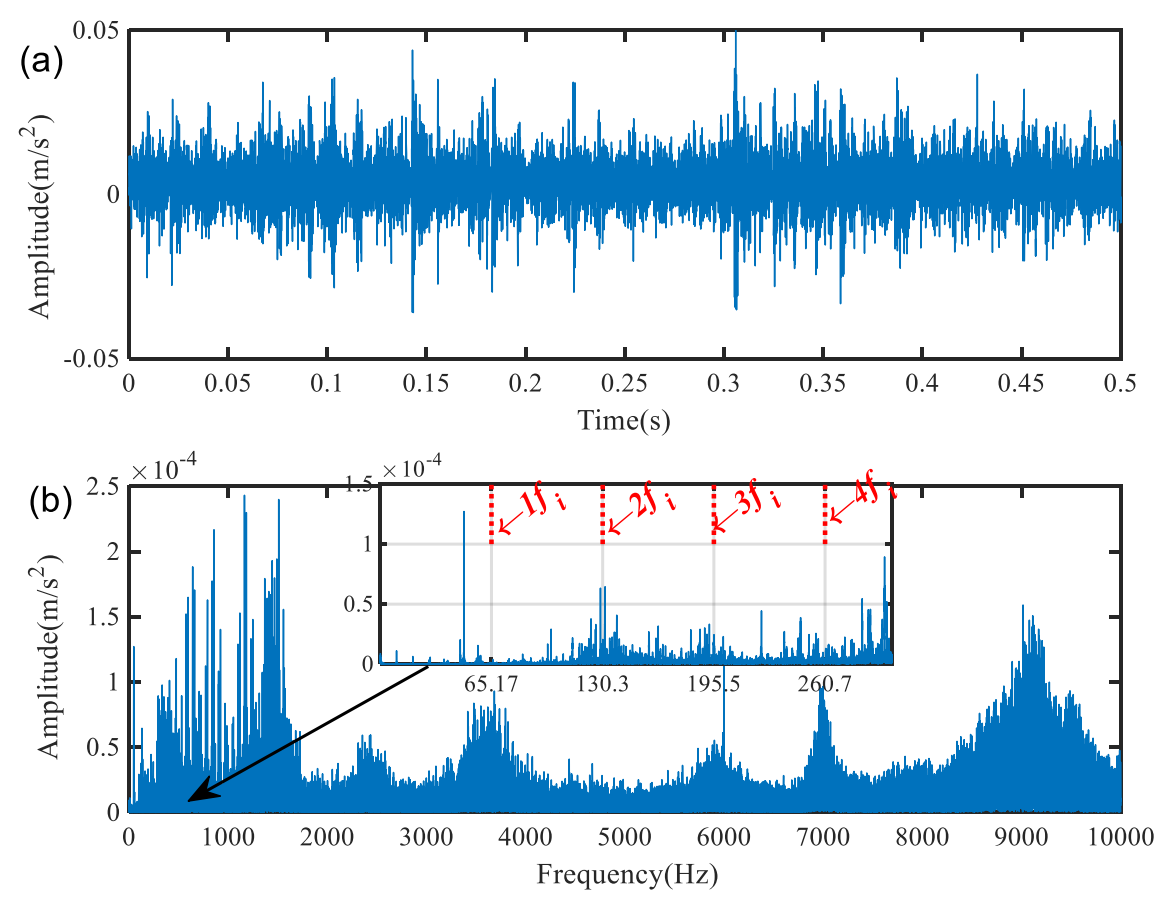

Fig. 32. Raw date of the faulty bearing in planetary gearbox: (a) waveform; (b) spectrum.

On the basis of the WPD-MSB, the measured signal presented in Fig. 32(a) was decomposed into 32 time-frequency subspaces using the Meyer wavelet, and the threshold criterion $F_{m}$ was then exploited to select the optimal threshold value $\Delta_{m}^{o}$ for WPD. The result of the threshold value optimization was shown in Fig. 33. It can be observed that there is an optimal threshold value $\Delta_{m}^{o}=$ 0.0071 as shown in Fig. 33. Subsequently, the NWPE matrix of denoising subspaces was calculated, and the proportional histogram of the NWPE distribution of the planetary gearbox bearing was depicted in Fig. 34. It presents the NWPE at nodes [5,0], [5,5] and [5,6] can be chose as the master subspace of the faulty bearing signal. Then, the master subspaces were used to obtain the fault related signals, and the result was depicted in Fig. 35. The envelope was then applied to the reconstructed signal, and its spectrum was displayed in Fig. 36. It is evident that the result is mixed with much random noise, and the effect of deterministic interference frequencies still exist with higher energy. 


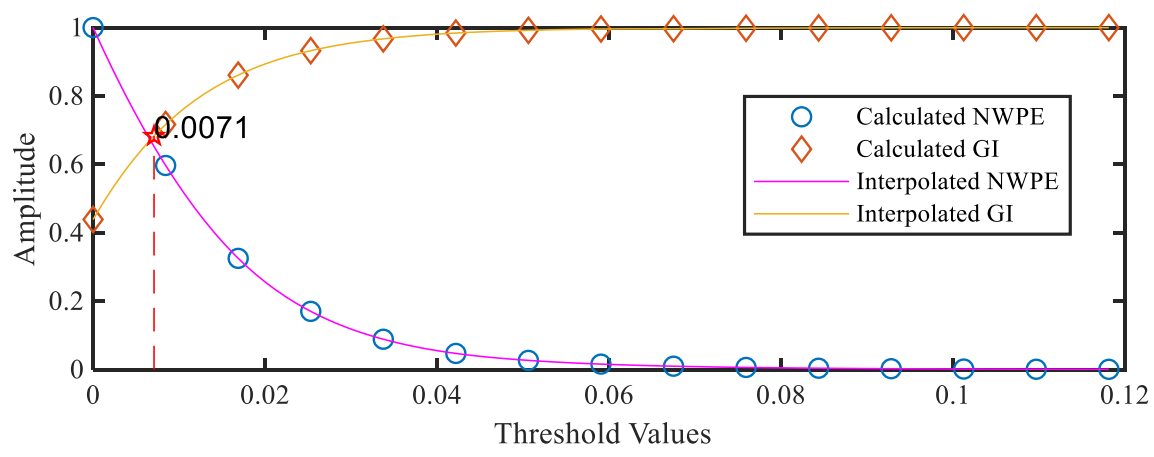

Fig. 33. Result of the threshold value optimization.

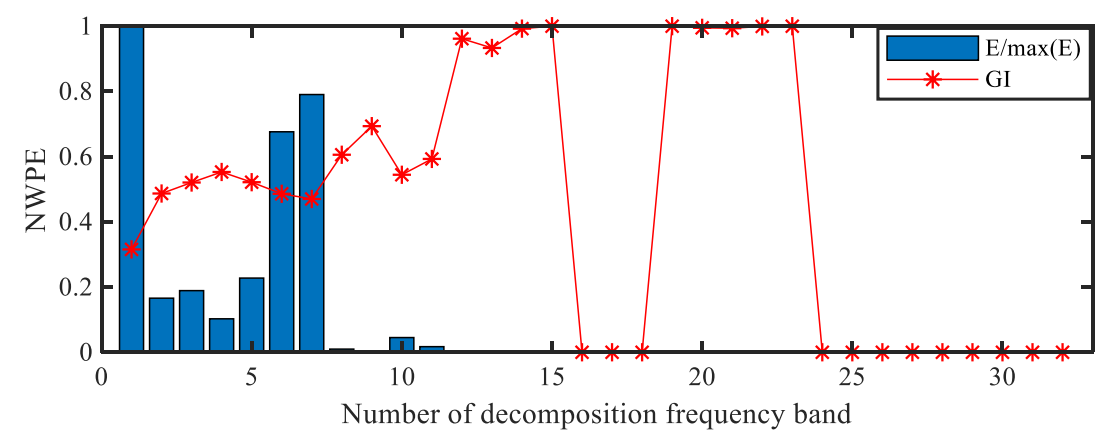

Fig. 34. Proportional histogram of the NWPE distribution.

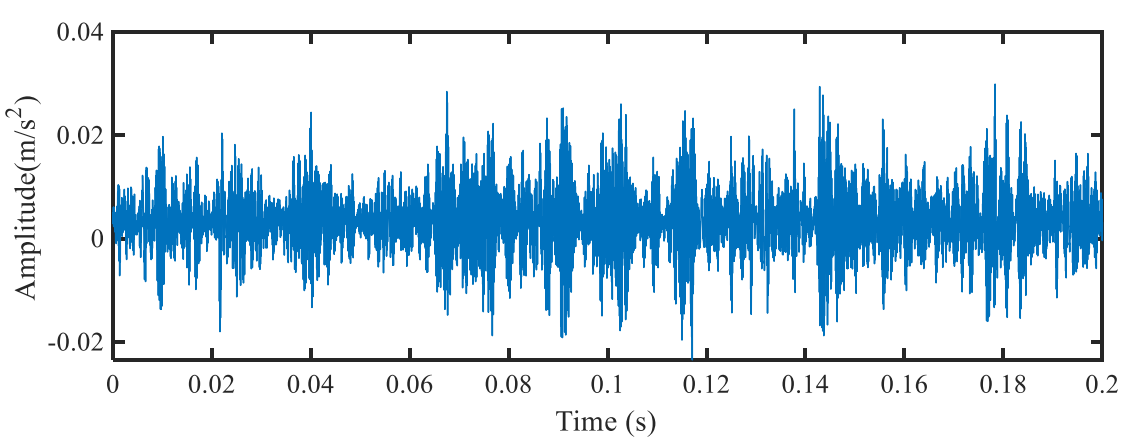

Fig. 35. Waveform of the reconstructed signal.

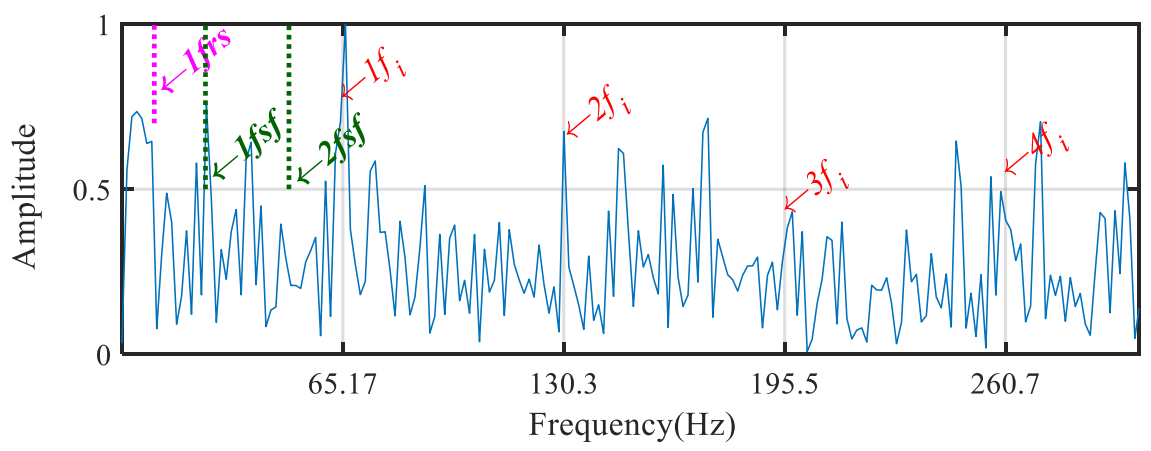

Fig. 36. Envelope spectrum of the reconstructed signal.

The MSB was applied to process the reconstructed signal instead of envelope analysis. In [35], the modulation signal bicoherence $(\mathrm{MSBc})$ was utilized to determine the intensity of the modulation component in the resonance frequency band, and the frequency band higher than $6 \mathrm{kHz}$ was excluded 
from the calculation of the MSB. The suboptimal MSB slices occur at 5 with maximum CFIC value as shown in Fig. 37. The MSB detector is obtained using the average the 5 suboptimal slices to extract the fault defect frequencies from the reconstructed signal. The analysis results using the WPD-MSB can be obtained and presented in Fig. 38. It can be observed that the random noise is nearly filtered out, and inner race fault frequency $f_{i}$ and its harmonics are recognized. This signifies that the defect on the planetary gearbox bearing can be accurately obtained by employing the WPD-MSB. In addition, small peaks appear at the sun gear rotational frequency $f_{r s}$ and its harmonics, the sun gear fault frequency $f_{s f}$ and its harmonics can also be identified in Fig. 38. The occurrence of these peaks may be due to manufacturing accuracy or improper installation.

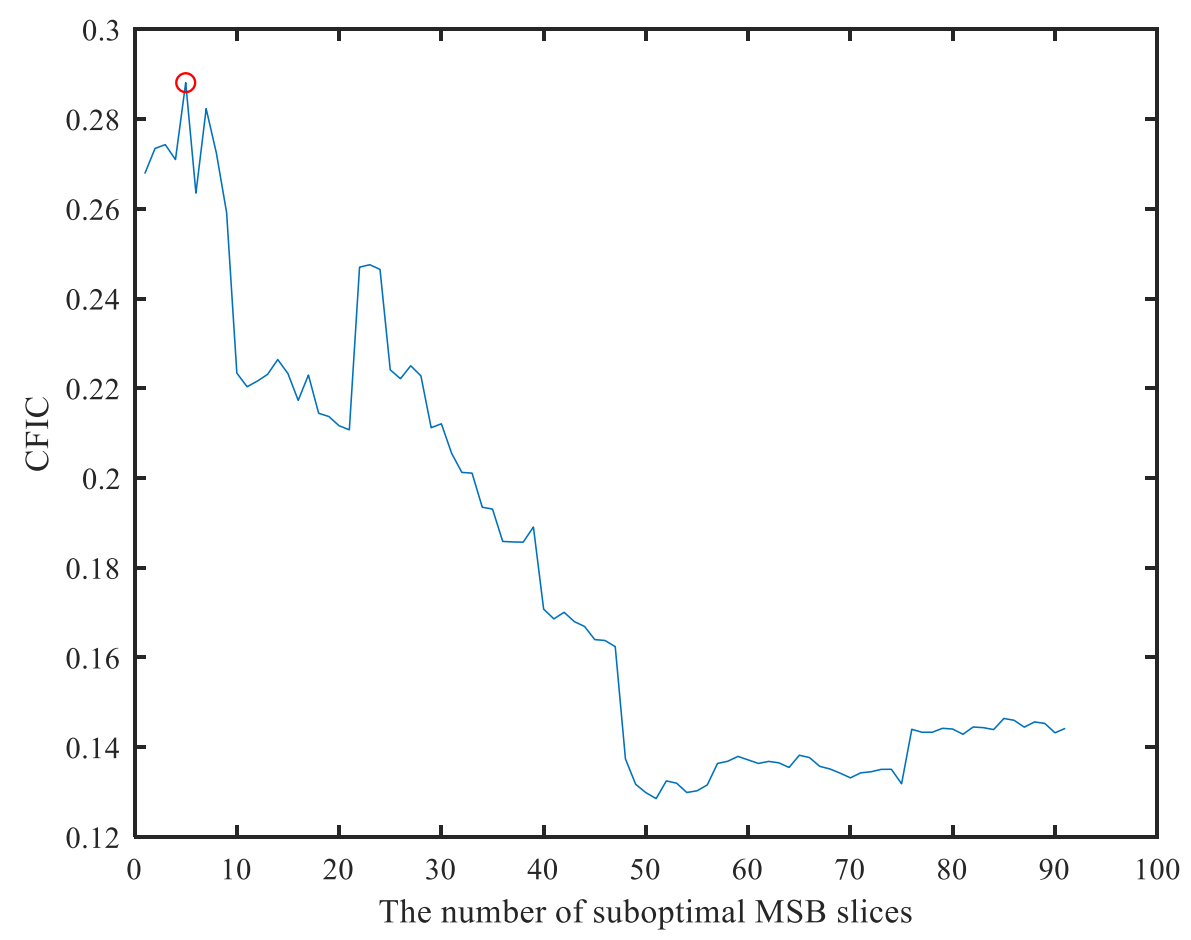

Fig. 37. CFIC value of suboptimal MSB slices. 

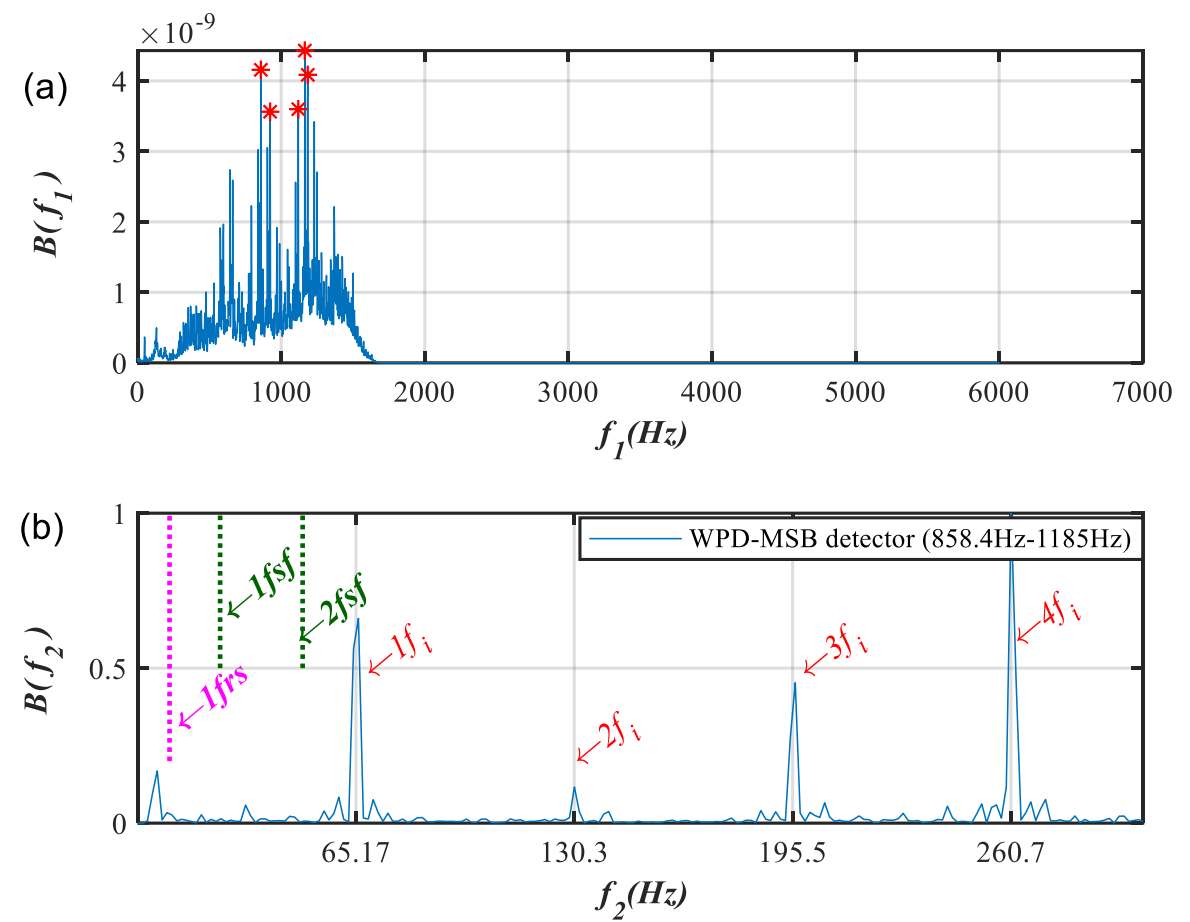

Fig. 38. Results of WPD-MSB: (a) WPD-MSB slices; (b) WPD-MSB detector.

As a comparison, the measured signal was also processed by the VMD-TEO and FK. The decomposition results of the VMD and its spectrum were depicted in Fig. 39. Compared with other IMFs, the frequency band of IMF1 was consistent with the resonance frequency band of the planetary gearbox bearing vibration signal. The TEO was then applied to the IMF1 to obtain fault defect frequencies, and its spectrum was depicted in Fig. 40. As displayed in Fig. 40(b), the sun gear rotational frequency $f_{r s}$ and its harmonics, the inner race fault frequency $f_{i}$ and its harmonics can be effectively extracted. However, the random noise and interference frequencies are not suppressed effectively. Moreover, the sun gear fault frequency $f_{s f}$ and its harmonics cannot be accurately obtained. Additionally, the detection results of the FK are depicted in Fig. 41. It found that the bandwidth and central frequency of the band pass filter are $1500 \mathrm{~Hz}$ and $5250 \mathrm{~Hz}$ in Fig. 41(a), respectively. The envelope and squared envelope spectrum of the filtered signal by FK are illustrated in Fig. 41(b) and (c). In Fig. 41(c), although the shaft rotational frequency $f_{r s}$, the sun gear fault frequency $f_{s f}$ and its harmonics can be identified, the irrelevant noise components in inner race fault frequency $f_{i}$ and its harmonics will directly influence the accuracy of the fault detection. 

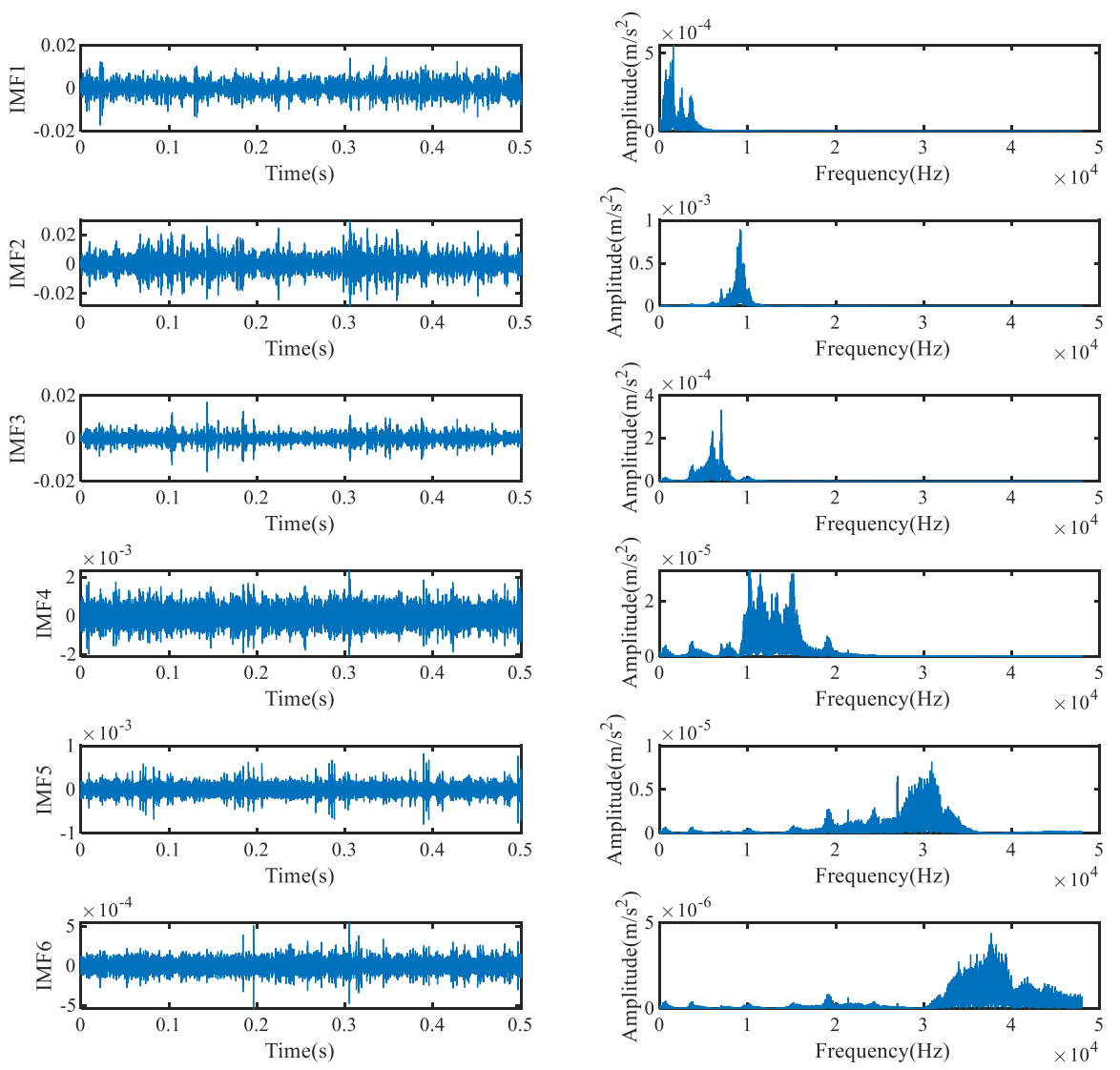

Fig. 39. Analysis results of the planetary gearbox bearing signal by VMD: (a) waveform; (b) spectrum.
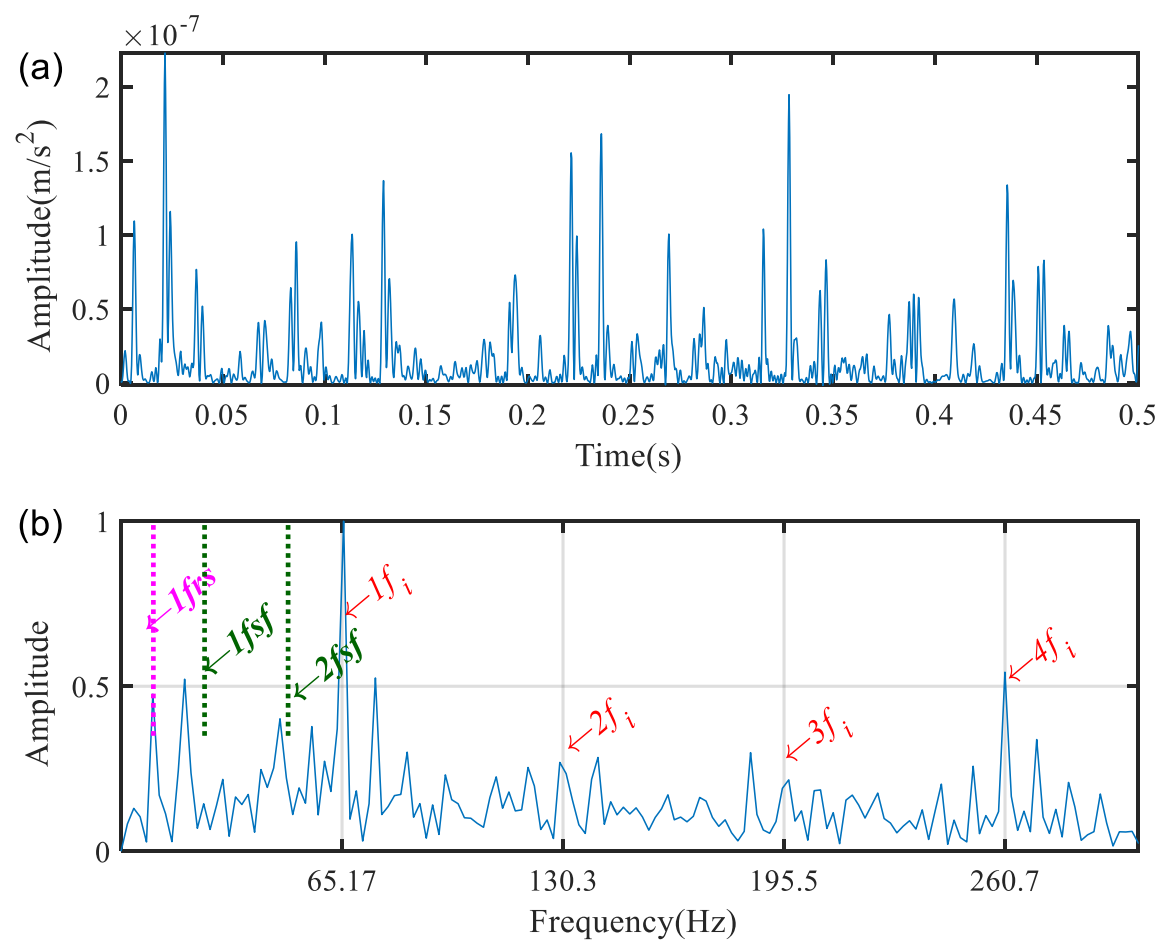

Fig. 40. Results of VMD-TEO: (a) waveform; (b) spectrum with central frequency of $1000 \mathrm{~Hz}$ and a bandwidth of $600 \mathrm{~Hz}$. 

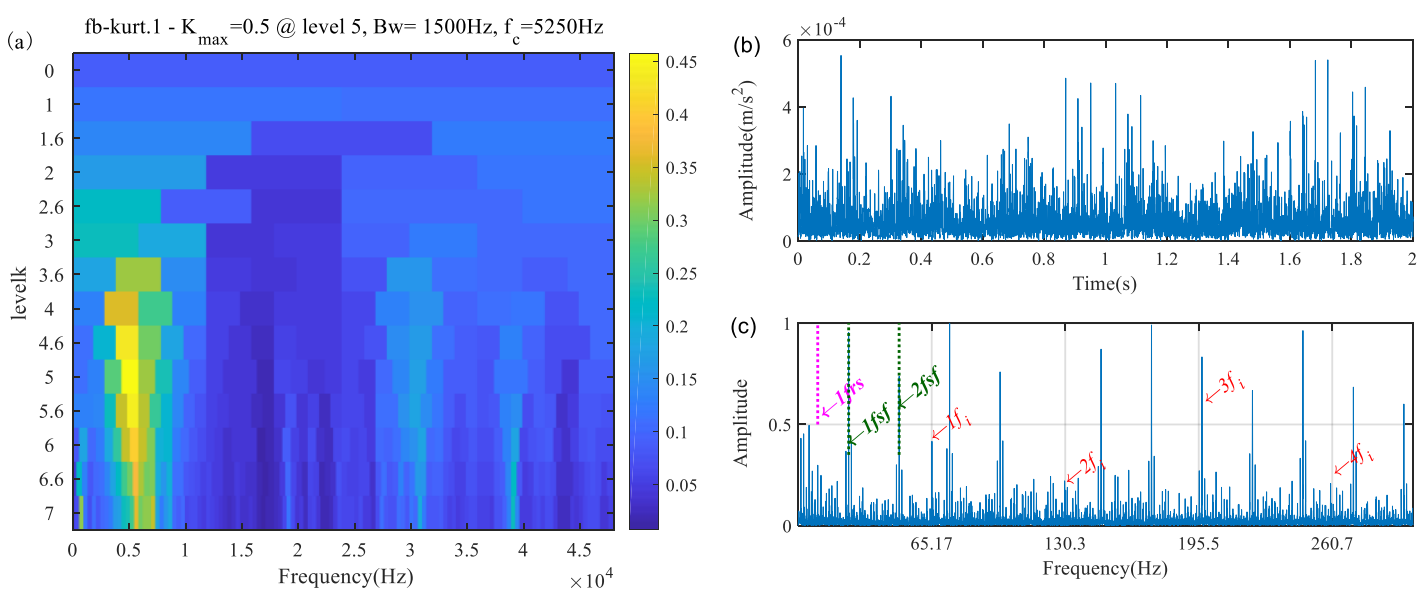

Fig. 41. Results of FK: (a) kurtogram; (b) envelope; (c) squared envelope spectrum.

Moreover, the conventional MSB was employed to handle the measured signal and the analysis result was depicted in Fig. 42. The result demonstrated that the conventional MSB can reflect the inner race fault frequency $f_{i}$ and its harmonics, but the random noise and interference frequencies are higher than that of the detection results acquired using the WPD-MSB as presented in Fig. 38. Table 5 depictes the fault defect index of the four algorithms. As illustrated in Table 5, compared with VMD-TEO, FK and MSB, the proposed WPD-MSB has excellent fault feature extraction performance. In conclusion, the comparison analysis indicated that the fault defect frequencies can be effectively identified and the modulation effects are enhanced using the proposed WPD-MSB method. In addition, the WPD-MSB can extract the planetary bearing fault characteristic frequencies more effectively than the VMD-TEO, FK and conventional MSB method.
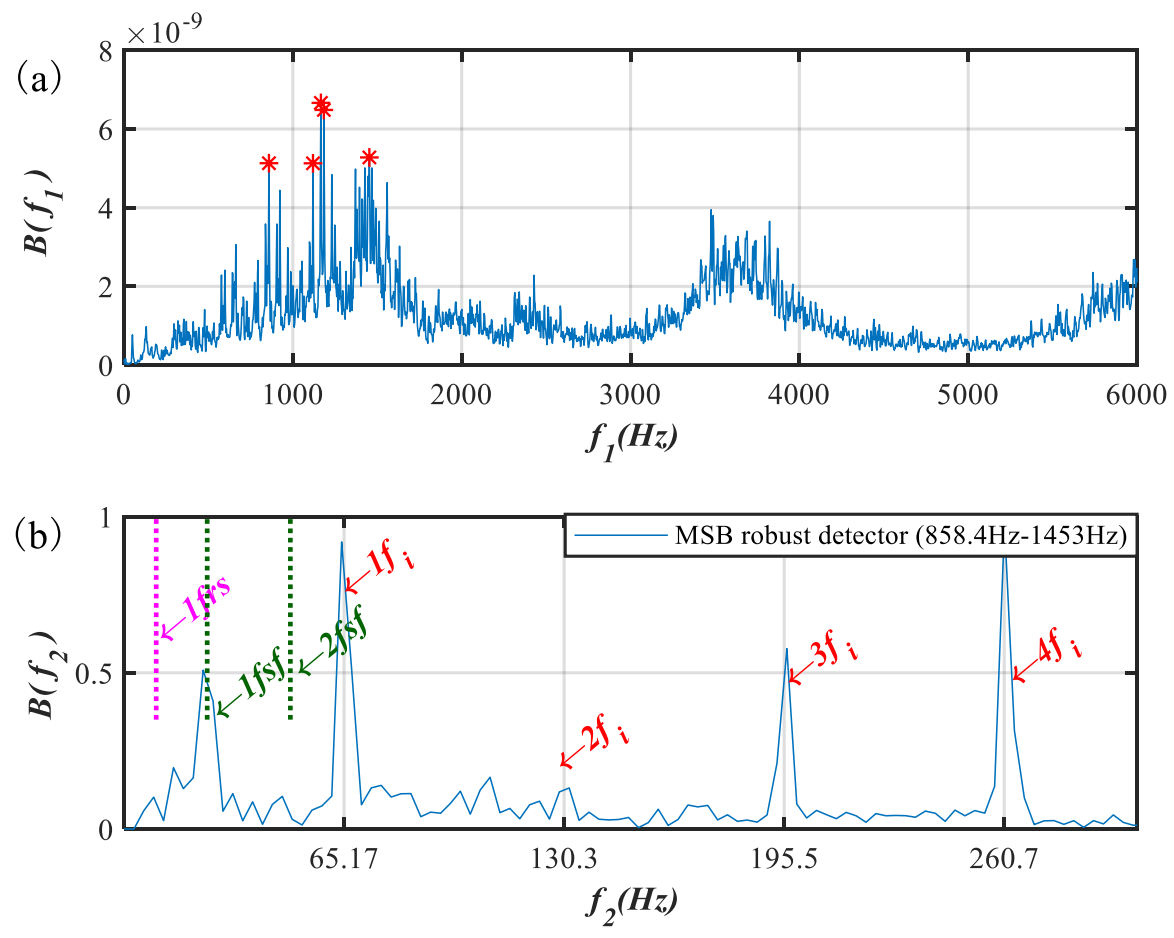

Fig. 42. Results of conventional MSB: (a) MSB slices; (b) MSB detector. 
Table 5. Fault defect index of the four algorithms.

\begin{tabular}{cc}
\hline Methods & Values \\
\hline WPD-MSB & 8.614 \\
VMD-TEO & 3.503 \\
FK & 0.744 \\
MSB & 8.160 \\
\hline
\end{tabular}

\section{Conclusion}

In this paper, a novel scheme based on an optimized WPD and MSB was proposed for rolling element bearing fault detection. The feasibility of the proposed scheme was verified by simulation and experimental studies. The analysis results prove that the proposed scheme achieves more satisfactory diagnostic results with higher accuracy. The main contributions of the proposed scheme are summarized as follows:

(1) A new threshold criterion is presented, which is applied to select the optimal threshold in WPD by considering the two indicators of unbiased autocorrelation of envelop and GI.

(2) The principle of GI is applied to select the master subspaces of WPT to obtain transient impulses.

(3) A criterion named CFIC index is developed to optimize the suboptimal MSB slices, which improves the performance of MSB in demodulating the modulation components.

(4) The analysis results prove that the proposed scheme is more competitive in improving the diagnosis accuracy than existing algorithms (e.g. VMD-TEO, FK and MSB). And the proposed technique provides a new perspective for improving bearing fault diagnosis.

\section{Funding}

The work is supported by the National Natural Science Foundation of China under Grant Nos. 51875166,51705127 and U1813222.

\section{References}

1. Feng Z, Chen X. Adaptive iterative generalized demodulation for nonstationary complex signal analysis: principle and application in rotating machinery fault diagnosis. Mech Syst Signal Pr 2018; 110: 1-27.

2. Li Y, Zuo MJ, Chen Z, et al. Railway bearing and cardan shaft fault diagnosis via an improved morphological filter. Struct Health Monit 2019; 133: 106303.

3. Zhang X, Liu Z, Miao Q, et al. An optimized time varying filtering based empirical mode decomposition method with grey wolf optimizer for machinery fault diagnosis. J Sound Vib 2018; 418: 55-78.

4. Yan X, Liu Y, Jia M. Health condition identification for rolling bearing using a multi-domain indicator-based optimized stacked denoising autoencoders. Struct Health Monit 2019; 134: 
106303.

5. Yang Z, Zhang J, Zhao Z, et al. Interpreting network knowledge with attention mechanism for bearing fault diagnosis. Appl Soft Comput 2020; 97: 106829.

6. Qu J, Zhang Z, Gong T. A novel intelligent method for mechanical fault diagnosis based on dual-tree complex wavelet packet transform and multiple classifier fusion. Neurocomputing 2016; 171: 837-853.

7. Hussein R, Shaban KB, El-Hag AH. Wavelet transform with histogram-based threshold estimation for online partial discharge signal denoising. IEEE Trans Instrum Meas 2015; 64 (12): 3601-3614.

8. Qin Y. A new family of model-based impulsive wavelets and their sparse representation for rolling bearing fault diagnosis. IEEE Trans Ind Electron 2018; 65 (3): 2716-2726.

9. Wang Z, Zhang Q, Xiong J, et al. Fault diagnosis of a rolling bearing using wavelet packet denoising and random forests. IEEE Sensors $J$ 2017; 17 (17): 5581-5588.

10. Xing Y, Wang Y, Shi L, et al. Sound quality recognition using optimal wavelet-packet transform and artificial neural network methods. Mech Syst Signal Pr 2016; 66-67: 875-892.

11. Liu H, Wang W, Xiang C, et al. A de-noising method using the improved wavelet threshold function based on noise variance estimation. Mech Syst Signal Pr 2018; 99: 30-46.

12. Hao $\mathrm{Q}$, Zhang $\mathrm{X}$, Wang $\mathrm{Y}$, et al. A novel rail defect detection method based on undecimated lifting wavelet packet transform and Shannon entropy-improved adaptive line enhancer. J Sound Vib 2018; 425: 208-220.

13. Xu X, Luo M, Tan Z, et al. Echo signal extraction method of laser radar based on improved singular value decomposition and wavelet threshold denoising. Infrared Phys Techn 2018; 92: $327-335$

14. Feng Z, Liang M, Chu F. Recent advances in time-frequency analysis methods for machinery fault diagnosis: A review with application examples. Mech Syst Signal Pr 2013; 38(1): 165-205.

15. Safara F, Doraisamy S, Azman A, et al. Multi-level basis selection of wavelet packet decomposition tree for heart sound classification. Comput Biol Med 2013; 43 (10): 1407-1414.

16. Choi S. Detection of valvular heart disorders using wavelet packet decomposition and support vector machine. Expert Syst Appl 2008; 35 (4): 1679-1687.

17. Wang J, He Q. Wavelet packet envelope manifold for fault diagnosis of rolling element bearings. IEEE T Instrum Meas 2016; 65 (11): 2515-2526.

18. Wang $\mathrm{Y}, \mathrm{Xu} \mathrm{G}$, Lin $\mathrm{L}$, et al. Detection of weak transient signals based on wavelet packet transform and manifold learning for rolling element bearing fault diagnosis. Mech Syst Signal Pr 2015; 54-55: 259-276.

19. Sadooghi MS, Khadem SE, A new performance evaluation scheme for jet engine vibration signal denoising. Mech Syst Signal Pr 2016; 76-77: 201-212. 
20. Jiang Y, Tang B, Qin Y, et al. Feature extraction method of wind turbine based on adaptive Morlet wavelet and SVD. Renew Energ 2011; 36(8): 2146-2153.

21. Sadegh HM, Esmaeilzadeh KS, Saleh SM. Quantitative diagnosis for bearing faults by improving ensemble empirical mode decomposition. ISA Trans 2018; 83: 261-275.

22. Lei $\mathrm{Y}$, Lin $\mathrm{J}, \mathrm{He} \mathrm{Z}$, et al. Application of an improved kurtogram method for fault diagnosis of rolling element bearings. Mech Syst Signal Pr 2011; 25: 1738-1749.

23. Tabrizi A, Garibaldi L, Fasana A, et al. Early damage detection of roller bearings using wavelet packet decomposition, ensemble empirical mode decomposition and support vector machine. Meccanica 2015; 50: 865-874.

24. Awal MA, Mostafa SS, Ahmad M, et al. An adaptive level dependent wavelet thresholding for ECG denoising. Biocybern Biomed Eng 2014; 34: 238-249.

25. Kong Y, Wang T, Chu F. Meshing frequency modulation assisted empirical wavelet transform for fault diagnosis of wind turbine planetary ring gear. Renew Energy 2019; 132: 1373-1388.

26. Donoho DL, Johnstone IM. Ideal spatial adaptation by wavelet shrinkage. Biometrika 1994; 81: 425-455.

27. Zhang X. Thresholding neural network for adaptive noise reduction. IEEE Trans Neural Networks $2001 ; 12: 567-584$.

28. Nasri M. Nezamabadi-Pour H. Image denoising in the wavelet domain using a new adaptive thresholding function. Neurocomputing 2009; 72: 1012-1025.

29. Wang X, Gu X, Chen BW. Image denoising based on improved wavelet threshold function for wireless camera networks and transmissions. Int J Distrib S 2015; 2: 23.

30. Mishra C, Samantaray AK, Chakraborty G. Rolling element bearing fault diagnosis under slow speed operation using wavelet de-noising. Measurement 2017; 103: 77-86.

31. Liu H, Wang W, Xiang C, et al. A de-noising method using the improved wavelet threshold function based on noise variance estimation. Mech Syst Signal Pr 2018; 99: 30-46.

32. Xu X. Single pulse threshold detection method with lifting wavelet denoising based on modified particle swarm optimization. Infrared Phys Techn 2018; 88: 174-183.

33. Han X, Chang X. An intelligent noise reduction method for chaotic signals based on genetic algorithms and lifting wavelet transforms. Inform Sci 2013; 218: 103-118.

34. Zhang R, Gu F, Mansaf H, et al. Gear wear monitoring by modulation signal bispectrum based on motor current signal analysis. Mech Syst Signal Pr 2017; 94: 202-213.

35. Tian X, Gu J, Rehab I, et al. A robust detector for rolling element bearing condition monitoring based on the modulation signal bispectrum and its performance evaluation against the Kurtogram. Mech Syst Signal Pr 2018; 100: 167-187.

36. Guo J, Zhen D, Li H, et al. Fault detection for planetary gearbox based on an enhanced average 
filter and modulation signal bispectrum analysis. ISA Trans 2020; 101: 408-420.

37. Guo J, Zhang H, Zhen D, et al. An enhanced modulation signal bispecturm analysis for bearing fault detection based on non-Gaussian noise suppression. Measurement 2020; 151: 107240.

38. $\mathrm{Xu} \mathrm{Y,} \mathrm{Fu} \mathrm{C,} \mathrm{Hu} \mathrm{N,} \mathrm{et} \mathrm{al.} \mathrm{A} \mathrm{phase} \mathrm{linearisation-based} \mathrm{modulation} \mathrm{signal} \mathrm{bispectrum} \mathrm{for} \mathrm{anlaysing}$ cyclostationary bearing signals. Struct Health Monit 2020; Epub ahead of print 23 August 2020. DOI: $10.1177 / 1475921720949827$.

39. He Q. Vibration signal classification by wavelet packet energy flow manifold learning. J Sound Vib 2013; 332: 1881-1894.

40. Guo J, Shi Z, Li H, et al. Early fault diagnosis for planetary gearbox based wavelet packet energy and modulation signal bispectrum analysis. Sensors 2018; 18: 2908.

41. Singh J, Darpe AK, Singh SP. Rolling element bearing fault diagnosis based on Over-Complete rational dilation wavelet transform and auto-correlation of analytic energy operator. Mech Syst Signal Pr 2018; 100: 662-693.

42. $\mathrm{Xu} \mathrm{Y,} \mathrm{Zhen} \mathrm{D,} \mathrm{Gu} \mathrm{JX,} \mathrm{et} \mathrm{al.} \mathrm{Autocorrelated} \mathrm{Envelopes} \mathrm{for} \mathrm{early} \mathrm{fault} \mathrm{detection} \mathrm{of} \mathrm{rolling} \mathrm{bearings.}$ Mech Syst Signal Pr 2021; 146: 106990.

43. Moshrefzadeh A, Fasana A. The Autogram: An effective approach for selecting the optimal demodulation band in rolling element bearings diagnosis. Mech Syst Signal Pr 2018; 105 294-318.

44. Miao Y, Zhao M, Lin J. Improvement of kurtosis-guided-grams via Gini index for bearing fault feature identification. Meas Sci Technol 2017; 28: 1-14.

45. Wang Y, Yang L, Xiang J, et al. A hybrid approach to fault diagnosis of roller bearings under variable speed conditions. Meas Sci Technol 2017; 28: 125104.

46. Wang $\mathrm{J}, \mathrm{Xu} \mathrm{M}$, Zhang $\mathrm{C}$, et al. Online bearing clearance monitoring based on an accurate vibration analysis. Energies 2020; 13: 389.

47. Randall RB, Antoni J. Rolling element bearing diagnostics-A tutorial. Mech Syst Signal Pr 2011; 25: $485-520$.

48. Liang M, Soltani IB. An energy operator approach to joint application of amplitude and frequency-demodulations for bearing fault detection. Mech Syst Signal Pr 2010; 24: 1473-1494.

49. Wang D. Some further thoughts about spectral kurtosis, spectral L2/L1 norm, spectral smoothness index and spectral Gini index for characterizing repetitive transients. Mech Syst Signal Pr 2018; $108 ; 360-368$.

50. Beale $\mathrm{C}$, Niezrecki $\mathrm{C}$, Inalpolat $\mathrm{M}$. An adaptive wavelet packet denoising algorithm for enhanced active acoustic damage detection from wind turbine blades. Mech Syst Signal Pr 2018; 142: 106754 .

51. Zhen D, Guo J, Xu Y, et al. A novel fault detection method for rolling bearings based on 
non-stationary vibration signature analysis. Sensors 2019; 19(18): 3994.

52. Xu B, Zhou F, Li H, et al. Early fault feature extraction of bearings based on Teager energy operator and optimal VMD. ISA Trans 2019; 86: 249-265.

53. Antoni J. Fast computation of the Kurtogram for the detection of transient faults. Mech Syst Signal Pr 2007; 21: 108-124.

54. Cheng Y, Zhou N, Zhang W, et al. Application of an improved minimum entropy deconvolution method for railway rolling element bearing fault diagnosis. $J$ Sound Vib 2018; 425: 53-69. 